\title{
Senescence marker protein 30 inhibits tumor growth by reducing HDAC4 expression in non-small cell lung cancer
}

\author{
Changjian Shao ${ }^{1,2 \#}$, Kai Guo ${ }^{1,3,4 \#}$, Liqun Xu ${ }^{2,5 \#}$, Yimeng Zhang ${ }^{6 \#}$, Hongtao Duan ${ }^{1}$, Yingtong Feng ${ }^{1,7,8}$, \\ Minghong Pan ${ }^{1}$, Di Lu ${ }^{2}$, Xiaoya Ren ${ }^{1}$, Apar Kishor Ganti', Taiki Hakozaki ${ }^{10}$, Jing Han ${ }^{6}$, Xiaolong Yan ${ }^{1}$, \\ Zhiqiang $\mathrm{Ma}^{2}$
}

${ }^{1}$ Department of Thoracic Surgery, Tangdu Hospital, The Fourth Military Medical University, Xi'an, China; ${ }^{2}$ Department of Medical Oncology, Senior Department of Oncology, The Fifth Medical Center of PLA General Hospital, Beijing, China; ${ }^{3}$ Department of Thoracic Surgery, Shaanxi Provincial People's Hospital, Xi'an, China; ${ }^{4}$ The Third Affiliated Hospital of Xi'an Jiaotong University, Xi'an, China; ${ }^{5}$ Department of Aerospace Medicine, The Fourth Military Medical University, Xi'an, China; ${ }^{6}$ Department of Ophthalmology, Tangdu Hospital, The Fourth Military Medical University, Xi'an, China; ${ }^{7}$ Department of Cardiothoracic Surgery, the 71th Group Army Hospital of PLA, Xuzhou, China; ${ }^{8}$ The Affiliated Huaihai Hospital of Xuzhou Medical University, Xuzhou, China; ${ }^{9}$ Division of Oncology-Hematology, Department of Internal Medicine, VA Nebraska Western Iowa Health Care System and University of Nebraska Medical Center, Omaha, NE, USA; ${ }^{10}$ Department of Thoracic Oncology and Respiratory Medicine, Tokyo Metropolitan Cancer and Infectious Diseases Center of Komagome Hospital, Tokyo, Japan

Contributions: (I) Conception and design: C Shao, K Guo, Z Ma, X Yan, J Han; (II) Administrative support: X Yan, Z Ma, J Han, AK Ganti, T Hakozaki; (III) Provision of study materials or patients: H Duan, D Lu, X Ren; (IV) Collection and assembly of data: K Guo, Y Zhang, M Pan, Y Feng; (V) Data analysis and interpretation: L Xu, Z Ma, C Shao; (VI) Manuscript writing: All authors; (VII) Final approval of manuscript: All authors.

"These authors contributed equally to this work.

Correspondence to: Xiaolong Yan, MD, PhD. Department of Thoracic Surgery, Tangdu Hospital, The Fourth Military Medical University, 1 Xinsi Road, Xi'an 710038, China. Email: yanxiaolong@fmmu.edu.cn; Jing Han, MD, PhD. Department of Ophthalmology, Tangdu Hospital, The Fourth Military Medical University, 1 Xinsi Road, Xi'an 710038, China. Email: hanjing.cn@163.com; Zhiqiang Ma, MD, PhD. Department of Medical Oncology, Senior Department of Oncology, The Fifth Medical Center of PLA General Hospital, 8 Dongdajie Road, Beijing 100071, China. Email: mzqfmmu@163.com.

Background: Senescence marker protein 30 (SMP30), which plays a pivotal role as a suppressor protein in cell proliferation, among other regulatory actions, is a marker of aging that shows decreased expression during senescence. Decreased SMP30 has been identified in several human cancers, but its expression and role in human non-small cell lung cancer (NSCLC) remain unclear.

Methods: Using tumor tissue and matched adjacent normal tissue from 341 patients with resected NSCLC, we assessed SMP30 expression using immunohistochemical methods. The relationship between SMP30 expression and clinicopathologic characteristics was investigated by Kaplan-Meier survival analysis and multivariate analysis. Cell viability assay, colony formation assay, EdU incorporation assay and in vivo tumor xenograft models were also performed to investigate NSCLC cell proliferation using A549 and H1299 cell lines. Recombinant lentivirus-meditated in vivo gene overexpression and Western blot were performed to clarify the underlying molecular mechanism of SMP30 inhibiting NSCLC proliferation.

Results: SMP30 expression was frequently downregulated in NSCLC tissue, as compared with adjacent non-tumor tissue. Kaplan-Meier survival analyses revealed NSCLC patients with low SMP30 expression had a significantly worse overall survival (OS), with median OS of 18 vs. 67 months in high SMP30 expression group. SMP30 overexpression significantly inhibited A549 and H1299 cell proliferation both in vitro and in tumor xenografts and downregulated the expression of $c-M y c$ and CyclinD1 protein. Moreover, Western blot analyses confirmed that SMP30 overexpression significantly inhibited the histone deacetylase 4 (HDAC4) level in NSCLC cells, and HDAC4 overexpression reversed SMP30-mediated NSCLC repression both in vitro and in vivo.

Conclusions: SMP30 inhibited NSCLC proliferation by reducing HDAC4 expression, and SMP30 and 
HDAC4 may serve as new prognostic biomarkers and future therapeutic targets for NSCLC.

Keywords: Senescence marker protein 30 (SMP30); proliferation; histone deacetylase 4 (HDAC4); non-small cell lung cancer (NSCLC)

Submitted Oct 26, 2021. Accepted for publication Dec 20, 2021.

doi: $10.21037 /$ tlcr-21-982

View this article at: https://dx.doi.org/10.21037/tlcr-21-982

\section{Introduction}

Lung cancer remains the leading cause of cancer-related deaths worldwide, and non-small-cell lung cancer (NSCLC), mainly consisting of lung squamous cell carcinoma (LUSC) and lung adenocarcinoma (LUAD), is the predominant type, accounting for approximately $85 \%$ (1). Despite great progress in therapeutic regimens, including surgical resection, chemotherapy, radiation therapy, immunotherapy, and targeted biological agents, the prognosis of NSCLC patients remains poor, with a 5 -year survival rate of approximately $26 \%(2,3)$. Clearly, there is a desperate need of novel diagnostic and therapeutic targets that can promote better evaluation of prognosis and optimization of treatments for individual NSCLC patients. Recent studies reported that senescence marker protein 30 (SMP30), a calcium-binding protein, may function as a tumor suppressor against tumor development and progression $(4,5)$.

SMP30, also known as regucalcin, shows decreased expression during senescence and is a marker of aging (6). $S M P 30$ was reported to be associated with aging and partial loss of hepatology function (7) and is involved in the regulation of intracellular $\mathrm{Ca}^{2+}$ levels, modulation of several cellular signaling pathways, and has antioxidant properties (8). Decreased levels of SMP30 leads to increased generation of reactive oxygen species, regarded as the most influential factor causing senescence (9). SMP30 has a protective action against oxidative damage through modulation of the activity of enzymes involved in generation of oxidative stress, as well as in antioxidant defense (9).

SMP30 plays a pivotal role as a suppressor protein in cell proliferation $(4,5)$. It has been demonstrated that SMP30 overexpression (OE) suppressed cell proliferation via decreasing DNA synthesis (10-12). Additionally, OE of SMP30 upregulated $\mathrm{p} 53$ and $\mathrm{p} 21$, while downregulating the mRNA expression of $c-M y c$ and H-ras, suggesting that SMP30 inhibits cell proliferation by modulating the expression of pro-oncogenes and tumor suppressor genes $(13,14)$. The expression of c-Jun and chk2 cell-cycle regulators was decreased in SMP30-transfected NRK52e cells (11). Interestingly, accumulating evidence demonstrates that suppression of SMP30 expression is involved in carcinogenesis $(4,15,16)$. Decreased SMP30 expression has been found in several human cancers, such as pancreatic cancer, breast cancer, and liver cancer $(5,17,18)$. Moreover, low SMP30 level was also associated with poor prognosis in these cancers $(5,16)$. However, SMP30 expression and its role in NSCLC are still unknown.

HDAC4 is a member of the class IIa HDAC family and is associated with a wide range of cellular and epigenetic processes as a transcriptional corepressor, similar to other members of the family (HDAC5, HDAC7, HDAC9) (19). It was reported that the OE of $H D A C 4$ promotes proliferation, metastasis and invasion of gastric cancer (20), esophageal carcinoma (21) and glioma (22). Moreover, elevated HDAC4 expression also correlated with poor prognosis $(21,23,24)$. HDAC4 was also reported to promote progression of lung cancer by regulating epithelial mesenchymal transition, autophagy and apoptosis $(25,26)$. Additionally, HDAC inhibitors were reported to exert growth arrest and apoptosis effect on tumor cells (27) and several HDAC inhibitors were used in NSCLC $(28,29)$. However, the oncostatic actions of HDAC4 and its detailed underlying molecular mechanisms in NSCLC remain largely unclear.

In the current study, we built the SMP30 OE NSCLC cell lines to investigate the actions of SMP30 on NSCLC proliferation both in vitro and in vivo. Western blot results revealed that $S M P 30$ OE dramatically affected $H D A C 4$ expression. Then we evaluated the role of $H D A C 4$ in SMP30-mediated tumor proliferation. Furthermore, the expression of SMP30 in 341 paired human NSCLC tissues and its correlation with related clinicopathologic features and survival were analyzed. We also analyzed whether the expressions of SMP30 could serve as predictive marker for prognosis of NSCLC patients. We present the following 
article in accordance with the ARRIVE reporting checklist (available at https://dx.doi.org/10.21037/tlcr-21-982).

\section{Methods}

\section{Database-mining}

To evaluate the expression of SMP30 in NSCLC tissue and paired normal tissue, data from Oncomine database (https:// www.oncomine.org/, Human Genome U133 Plus 2.0 Array, Reporter ID: 210751_s_at) were analyzed. Correlation analysis of SMP30 expression and clinicopathologic parameters was also performed. Moreover, we also analyzed the associations between SMP30 expression and NSCLC patient prognosis using data from Kaplan-Meier plotter database (https://kmplot.com/, use earlier release of the database: all 2015 version, $n=2,437$ ) and The Human Protein Atlas database (the RNA-seq data based on The Cancer Genome Atlas, http://www.proteinatlas.org/).

\section{NSCLC tissue samples and tissue microarray immunobistochemistry (IHC)}

We included 341 patients who underwent NSCLC surgery at Tangdu Hospital between May 2009 and December 2013 in this retrospective study. Among them, 152 patients were of early clinical stage (stage I/II) and 189 patients were of late clinical stage (stage III/IV). None of the patients had received radiotherapy or chemotherapy before surgery, and the final follow-up was updated until death or December 2018, whichever came first. All procedures performed in this study involving human participants were in accordance with the Declaration of Helsinki (as revised in 2013). This study was approved by the Ethics Committee of the Fourth Military Medical University (No. TDLL202110-03) and informed consents were not required for this retrospective study. Using paraffin-embedded tissue microarray containing 341 pairs NSCLC and matched adjacent normal lung tissues, IHC staining was conducted using the primary antibodies of anti-SMP30 $(1: 50,17947$ 1-AP, Proteintech), and standard protocols were followed as previously described (30).

The IHC staining score was based on two criteria: (I) percentage of positive cells $(0, \leq 5 \%$; $1,6-25 \% ; 2,26-50 \%$; $3,51-75 \%$; and $4,>75 \%)$; (II) staining intensity $(0$, negative; 1 , yellow; 2 , brown; and $3, \tan )$. The two scores were multiplied to produce the total score and the median score was used to divide the NSCLC samples into those with low and high SMP30 expression.

\section{Cell culture and lentivirus (LV) infection}

Human NSCLC A549 (ATCC Cat\# CRM-CCL-185, RRID: CVCL_0023) and H1299 (ATCC Cat\# CRL5803, RRID: CVCL_0060) cell lines were purchased from the American Type Culture Collection (ATCC, VA, USA) in January 2018, and the A549 and H1299 cells were authenticated by the ATCC upon purchase (5 months prior to experimental research) using morphology, karyotyping, and PCR based approaches to profile the cytochrome C oxidase I gene (COI analysis) and short tandem repeat to confirm the identity of human cell lines and to rule out both intra- and interspecies contamination (see ATCC website https://www.atcc.org/CellAuthenticationMatters. aspx). Cells were cultured in DMEM with high glucose (Gibco, NY, USA), supplemented with $10 \%$ fetal bovine serum (Gibco), penicillin-streptomycin solution (100 units/mL; Solarbio, Beijing, China). SMP30, HDAC4 and paired empty vector LVs were purchased from Genechem (Shanghai, China). LV infection of A549 and H1299 cells was performed according to the Genechem protocols. Next, SMP30 and HDAC4 OE cells or negative controls (NCs) were selected with puromycin.

\section{Cell viability assay}

The A549 and H1299 cell viability assay used the CCK8 kit according to the manufacturer's instructions (7Sea, Shanghai, China). Optical density values were measured at $450 \mathrm{~nm}$ through a microplate reader (SpectraMax 190, Molecular Device, USA).

\section{Colony formation assay}

A total of 500 A549 and H1299 cells were seeded and cultured in 6-well plates for 12-14 days, after which the plates were photographed and colonies containing $>50$ cells were counted after staining with $0.1 \%$ crystal violet.

\section{5-Ethynyl-2'-deoxyuridine (EdU) incorporation assay}

Cell proliferation was assessed by EdU incorporation assay. A BeyoClick EdU Cell proliferation kit with Alexa Fluor 594 (Beyotime, Shanghai, China) was used according to the manufacturer's instructions. Cells were imaged by Olympus FV1000 confocal microscope (Olympus, Tokyo, Japan). 
EdU-positive cells were manually counted and expressed as the percentage of cells calculated from nuclear labeling with Hoechst 33342.

\section{In vivo tumor xenograft models}

Animal experiments were performed under a project license (No. IACUC-20210609) granted by the Animal Ethics Committee of the Fourth Military Medical University, in compliance with the institutional guidelines for the care and use of animals. A protocol was prepared before the study without registration. For each part, 5 athymic nude mice (male, 4-6 weeks, 18-20 g) were obtained from the university's Laboratory Animal Center. They were housed singly under the conditions of $12 \mathrm{~h} \mathrm{light} / 12 \mathrm{~h}$ dark cycle, 65 $75^{\circ} \mathrm{F}\left(18-23^{\circ} \mathrm{C}\right)$ and $40-60 \%$ humidity with adequate food and water. The left and right flanks of 5 nude mice (male, 4-6 weeks, 18-20 g) were subcutaneously inoculated with LV-control and LV-SMP30 cells separately while another 5 mice received LV-SMP3O + LV-HDAC4 and LV-SMP30 $+\mathrm{LV}$-control cells, respectively. Different groups of $5 \times 10^{6}$ H1299 cells were separately inoculated subcutaneously into the right or left flank of 6-week-old male nude mice for in vivo tumor xenograft assay. The tumor size and body weight were assessed every 3 days (the longer diameter of the tumor should not be $>20 \mathrm{~mm}$ ). Twenty-one to Twenty-eight days after injection, the animals were anesthetized and the tumors were excised for additional analysis before the humane death of the animal. After photographing and weighing the tumor, part of each sample was fixed in formalin, and another part was prepared for further analysis.

\section{Western blot}

Western blot was performed as previously described (31). Antibodies against $\operatorname{SMP} 30(1: 1,000,17947-1$-AP, Proteintech), against HDAC4 (1:1,000, Proteintech Cat\# 17449-1-AP, RRID: AB_2118864), anti-CyclinD1 (1:1,000, 60186-1-Ap, Proteintech), anti-c-Myc (1:1,000, \#5605, CST) anti- $\beta$-actin (1:5,000, ab6276, Abcam), and anti-tubulin (1:1,000, \#2148, CST) were used. The 1:5,000 dilution of horseradish-peroxidase-linked anti-mouse or rabbit IgG was used as the secondary antibody (Zhongshan Company, Beijing, China).

\section{Cell treatment}

The pan-HDAC inhibitor vorinostat (SAHA), and the specific class IIa inhibitor TMP269, were obtained from MedChemExpress and prior to the experiment were prepared in DMSO and diluted in culture media immediately. One day after seeding onto culture dish, normal cells and HDAC4 overexpressing cells were divided into four groups: (I) control group; (II) $0.625 \mu \mathrm{M}$ SAHA/20 $\mu \mathrm{M}$ TMP269 group; (III) $1.25 \mu \mathrm{M}$ SAHA/40 $\mu \mathrm{M}$ TMP269 group; (IV) $2.5 \mu \mathrm{M}$ SAHA/60 $\mu$ M TMP269 group. After the above treatments for $48 \mathrm{~h}$, cells were analyzed.

\section{Statistical analysis}

Data analyses were carried out with SPSS 23.0 (SPSS Inc., IL, USA) software. The relationships between SMP30 expression and the clinicopathologic parameters of the NSCLC patients were evaluated by $\chi^{2}$ test or Fisher's exact test. Survival analyses were examined by Kaplan-Meier method, then compared with the log-rank test. Univariate and multivariate survival analyses were conducted using Cox hazards regression models. Results were compared between groups by Student's $t$-test. Data are presented as the mean \pm standard deviation (SD). $\mathrm{P}<0.05$ was considered as statistically significant.

\section{Results}

\section{Expression of SMP30 in NSCLC patients}

To investigate the expression of SMP30 in NSCLC, we initially conducted database-mining to compare the gene expression profiles of SMP30 between normal lung and cancer tissues. Oncomine database (https://www.oncomine.org/) analysis revealed that the $S M P 30 \mathrm{mRNA}$ level was significantly lower in various cancer types compared with normal tissues, but especially in lung cancer (Figure S1A). Results showed that SMP30 mRNA levels in NSCLC, including large-cell lung carcinoma, LUAD and LUSC, were significantly decreased compared with normal lung tissue samples (Figure S1B). To further validate the SMP30 expression in NSCLC tissues, we performed IHC analysis to detect the SMP30 level in a tissue microarray containing 341 paired tumor-normal lung tissues (Figure 1A) and it was clearly lower in NSCLC than in adjacent noncancerous lung samples (Figure 1B).

In order to characterize the roles of SMP30 in NSCLC, we analyzed whether its expression was associated with clinicopathologic variables (Table 1). Of the 341 samples, 133 had a score of 0 while two samples had a score of 12 . 
A

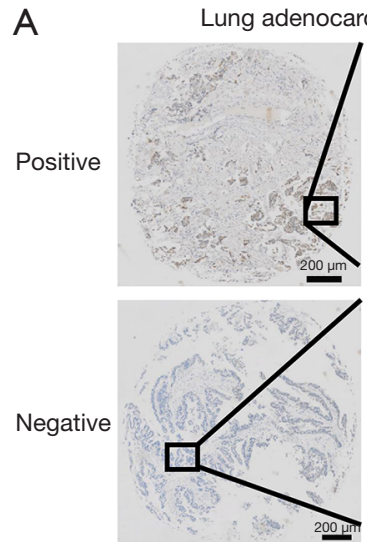

B
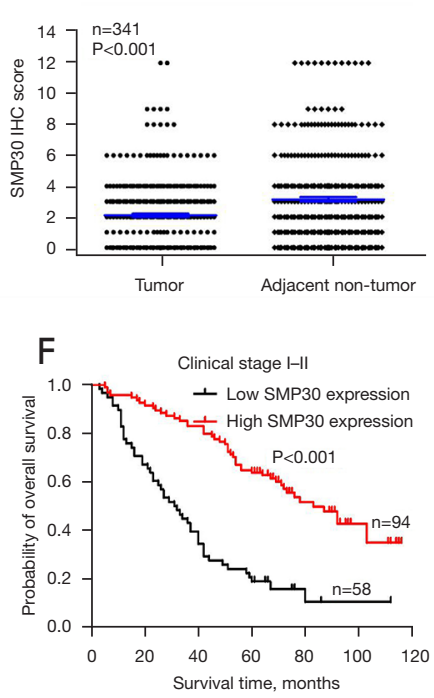

Squamous cell lung carcinoma tissue
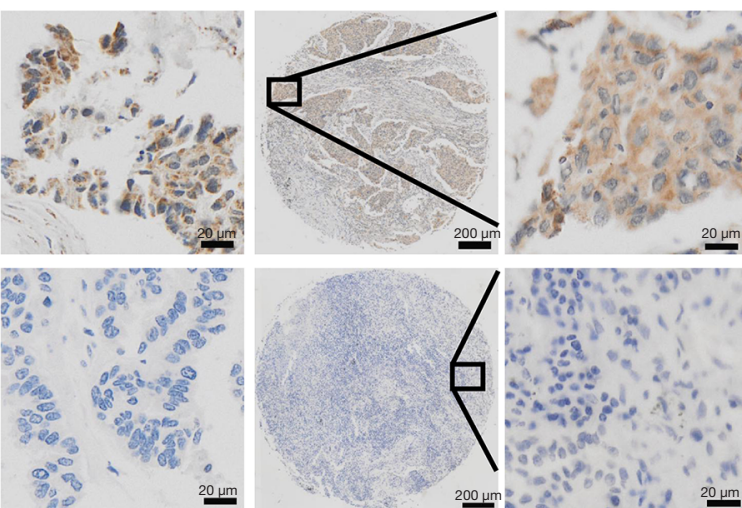

C
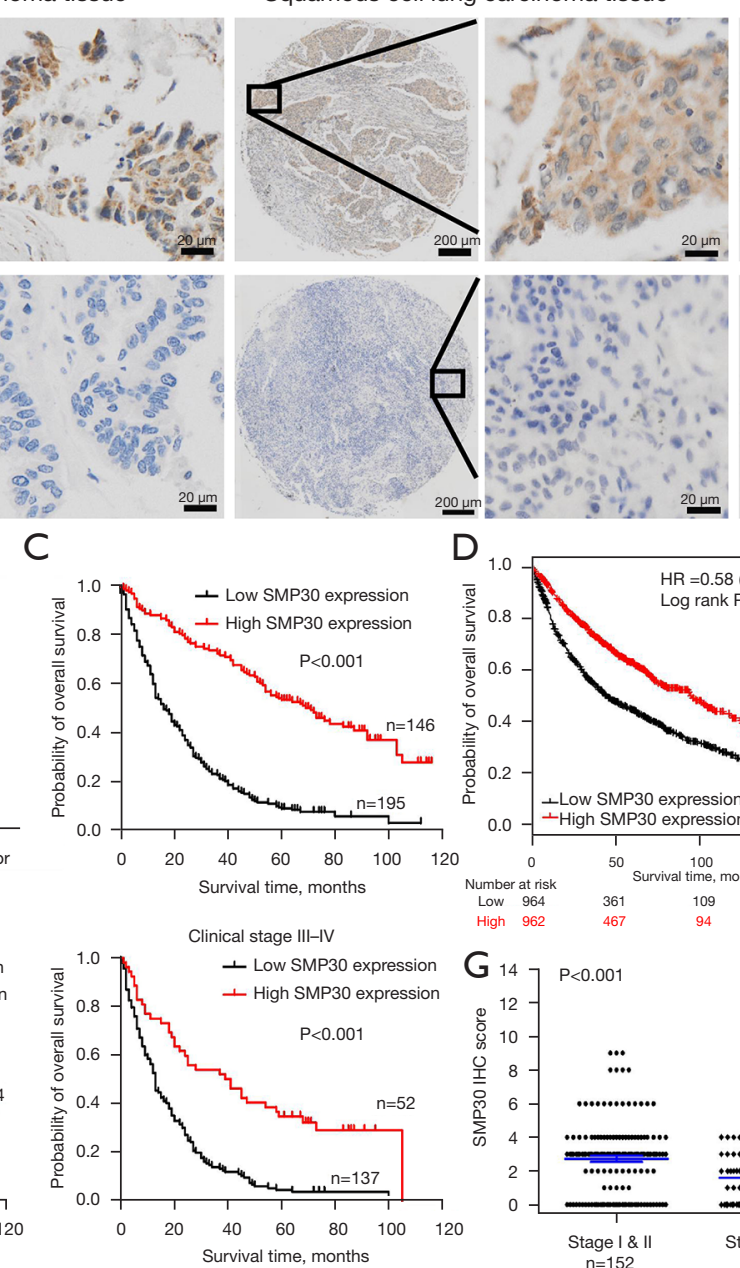
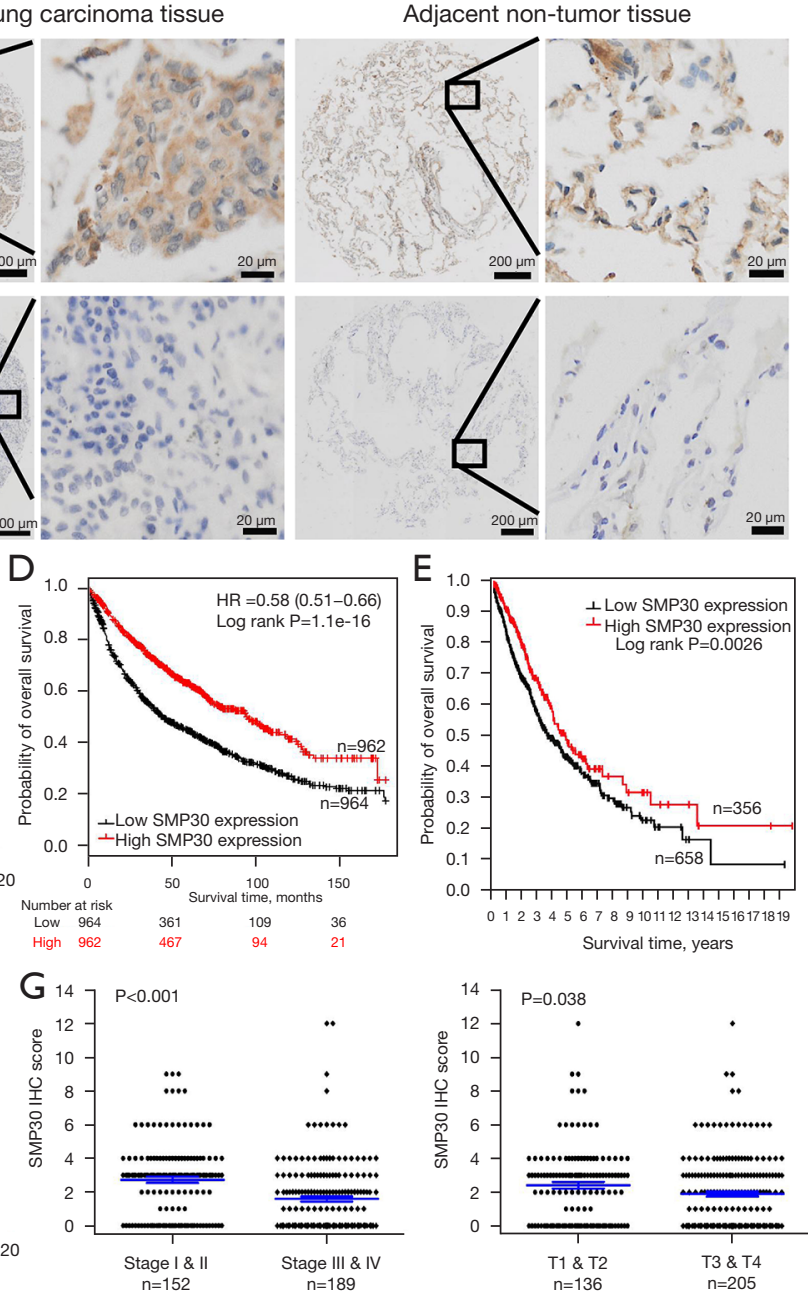

Figure 1 Correlation of low SMP30 level with worse overall survival in NSCLC patients. (A) Representative immunohistochemical (IHC) images for SMP30 expression in NSCLC (lung squamous cell carcinoma and lung adenocarcinoma) and adjacent noncancerous tissues. Scale bar, 200 and $20 \mu \mathrm{m}$ (inset) respectively. (B) Statistical analysis of SMP30 expression in tumor tissue and adjacent noncancerous tissue of 341 NSCLC patients through IHC staining. (C-F) Kaplan-Meier survival analyses of high/low SMP30 expression based on tissue microarray IHC results for 341 NSCLC patients (C), 1,926 NSCLC patients in the Kaplan-Meier plotter database (D), 1,014 NSCLC patients from The Human Protein Atlas (original RNA-seq data from TCGA) (E), 152 NSCLC patients in the early clinical stages, and 189 NSCLC patients in the late clinical stages (F). (G) Statistical analysis of SMP30 expression in different clinical stages and T classifications of 341 NSCLC patients through IHC staining. SMP30, senescence marker protein 30; NSCLC, non-small cell lung cancer; TCGA, The Cancer Genome Atlas.

The median score for the entire cohort was 2. On univariate analysis, SMP30 expression was significantly associated with tumor size, tumor invasion, lymphatic metastasis, distant metastasis, cancer differentiation and clinical stage respectively (Table 1). These results indicated that abnormal SMP30 expression was involved in NSCLC progression.

\section{Correlation of SMP30 expression and NSCLC prognosis and survival}

We analyzed the association between SMP30 expression and NSCLC patient prognosis using Kaplan-Meier analysis and log-rank test for significance estimates. The KaplanMeier survival curves showed that NSCLC patients with 
Table 1 Association of SMP30 expression with clinicopathologic parameters of patients with NSCLC

\begin{tabular}{|c|c|c|c|c|}
\hline \multirow{2}{*}{ Parameter } & \multirow{2}{*}{$\mathrm{N}$} & \multicolumn{3}{|c|}{ SMP30 expression } \\
\hline & & Low & High & $P$ value \\
\hline Age, years & & & & 0.911 \\
\hline$<60$ & 153 & 88 & 65 & \\
\hline$\geq 60$ & 188 & 107 & 81 & \\
\hline Sex & & & & 0.503 \\
\hline Male & 272 & 158 & 114 & \\
\hline Female & 69 & 37 & 32 & \\
\hline Tumor location & & & & 0.803 \\
\hline Left lung & 142 & 89 & 53 & \\
\hline Right lung & 199 & 106 & 93 & \\
\hline Tumor size, $\mathrm{cm}$ & & & & 0.003 \\
\hline$<5$ & 124 & 58 & 66 & \\
\hline$\geq 5$ & 217 & 137 & 80 & \\
\hline T classification & & & & 0.001 \\
\hline $\mathrm{T} 1$ & 6 & 3 & 3 & \\
\hline $\mathrm{T} 2$ & 130 & 62 & 68 & \\
\hline T3 & 127 & 71 & 56 & \\
\hline T4 & 78 & 59 & 19 & \\
\hline Lymph node metastases & & & & $<0.001$ \\
\hline No & 156 & 72 & 84 & \\
\hline N1-N3 & 185 & 123 & 62 & \\
\hline Metastasis & & & & 0.022 \\
\hline No & 330 & 185 & 145 & \\
\hline Yes & 11 & 10 & 1 & \\
\hline Differentiation & & & & $<0.001$ \\
\hline Well and moderate & 235 & 108 & 127 & \\
\hline Poorly and not & 106 & 87 & 19 & \\
\hline Clinical stage & & & & $<0.001$ \\
\hline I & 48 & 12 & 36 & \\
\hline II & 104 & 46 & 58 & \\
\hline III & 178 & 127 & 51 & \\
\hline IV & 11 & 10 & 1 & \\
\hline
\end{tabular}

SMP30, senescence marker protein 30; NSCLC, non-small cell lung cancer. low SMP30 expression were associated with worse overall survival (OS) (log-rank $\mathrm{P}<0.001$, Figure 1C). Consistently, the Kaplan-Meier plotter database (https://kmplot.com/) survival analysis based on 1,926 NSCLC cases suggested that a low SMP30 level was significantly correlated with poor prognosis [hazard ratio $(\mathrm{HR})=0.58, \log$-rank $\mathrm{P}<0.001$, Figure 1D]. Furthermore, we analyzed the survival of 1,014 NSCLC cases through The Human Protein Atlas database (the RNA-seq data based on The Cancer Genome Atlas, https://www.proteinatlas.org/), and verified that low $S M P 30$ level was related to poor prognosis (Figure 1E). Interestingly, we found that NSCLC patients with low SMP30 expression had worse OS when the patients were analyzed based on stage at diagnosis. This was evident in both early stage (stage I/II) and more advanced stage (stage III/IV) $(\mathrm{P}<0.001$, Figure $1 F)$. Our results also indicated that NSCLC patients with larger tumors (T3/T4) and high clinical stage (stage III/IV) had lower expression of SMP30 than those with smaller tumor invasion (T1/T2) and low clinical stage (stage I/II) $(\mathrm{P}<0.001$, Figure $1 G)$.

To further assess whether $S M P 30$ expression represents a prognostic factor for patients with NSCLC, we performed Cox proportional hazard regression analysis of the patients' OS. Univariate analysis showed that low SMP30 expression negatively correlated with NSCLC survival (Table 2). In the multivariate survival analysis, when other risk factors such as tumor invasion, lymphatic invasion, distant metastasis, cancer differentiation, and clinical stage were taken in consideration, the SMP30 expression was still associated with a lower risk of death 0.383 (95\% CI: 0.282-0.518, $\mathrm{P}<0.001)$.

\section{Low SMP30 level and poor prognosis in both LUAD and LUSC patients}

We further studied the prognostic role of SMP30 expression in LUAD and LUSC patients. The Kaplan-Meier plotter database survival analysis including 719 LUAD cases indicated that low SMP30 expression in LUAD patients was significantly associated with poor prognosis ( $\mathrm{HR}=0.44$, logrank $\mathrm{P}<0.001$, Figure $2 A$ ). Analysis of SMP30 expression in 131 LUAD patients in our cohort, verified that low SMP30 level significantly correlated with worse OS, with median OS in low SMP30 expression group and high SMP30 expression group was 22 and 47 months respectively (logrank $\mathrm{P}<0.001$, Figure $2 B$ ). Moreover, we found the prognosis of LUAD patients with low SMP30 expression was worse in both early clinical stage (stage I/II) (median OS of 
Table 2 Univariate and multivariate analyses of the correlation between clinicopathological variables for survival of patients with non-small cell lung cancer

\begin{tabular}{|c|c|c|c|c|c|c|c|}
\hline Variable & Category & \multicolumn{3}{|c|}{ Univariate analysis } & \multicolumn{3}{|c|}{ Multivariate analysis } \\
\hline Age & $<60 / \geq 60$ years & 1.171 & $0.919-1.491$ & 0.201 & - & - & - \\
\hline Sex & Male/female & 1.067 & $0.789-1.442$ & 0.675 & - & - & - \\
\hline Tumor location & Left lung/right lung & 0.879 & $0.689-1.121$ & 0.299 & - & - & - \\
\hline Lymphatic invasion & Yes/no & 1.740 & $1.359-2.229$ & $<0.001$ & 0.991 & $0.714-1.377$ & 0.959 \\
\hline Distant metastasis & Yes/no & 2.425 & $1.323-4.445$ & 0.004 & 1.171 & $0.537-2.553$ & 0.692 \\
\hline Differentiation & Well and moderate/poorly and not & 5.150 & $3.913-6.777$ & $<0.001$ & 3.272 & $2.452-4.365$ & $<0.001$ \\
\hline Clinical stage & I/II/III/IV & 1.549 & $1.332-1.800$ & $<0.001$ & 1.378 & $1.018-1.867$ & 0.038 \\
\hline
\end{tabular}
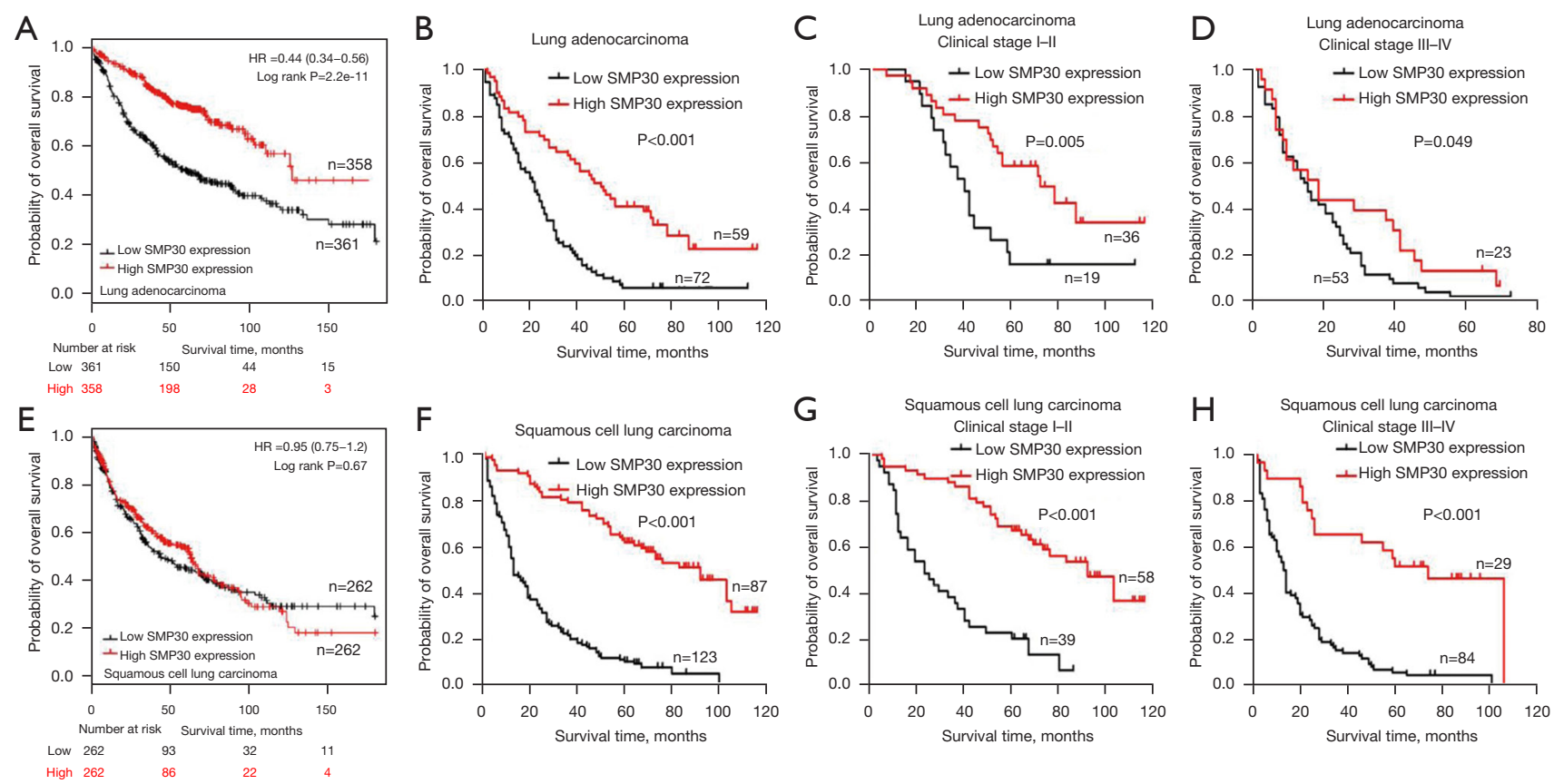

Figure 2 Correlation of low SMP30 level with worse overall survival in both LUAD and LUSC patients. (A,E) Kaplan-Meier survival analyses of high/low SMP30 expression in 719 LUAD patients and 524 LUSC patients, respectively, based on the Kaplan-Meier plotter database. (B-D,F-H) Kaplan-Meier survival analyses of high/low SMP30 expression in 131 LUAD patients and 210 LUSC patients based on the tissue microarray immunohistochemical results. SMP30, senescence marker protein 30; HR, hazard ratio; LUAD, lung adenocarcinoma; LUSC, lung squamous cell carcinoma. 
31 vs. 103 months) and late clinical stage (stage III/IV) (median OS of 13 vs. 39 months) subgroups (log-rank $\mathrm{P}<0.05$, Figure $2 C, 2 D$ ).

Interestingly, the Kaplan-Meier plotter database showed no significance between SMP30 expression and LUSC patients' $\mathrm{OS}(\mathrm{HR}=0.95, \log$-rank $\mathrm{P}=0.67)$ (Figure $2 E)$, but our Kaplan-Meier survival analysis based on 210 LUSC patients showed that low SMP30 level was associated with poor prognosis (median OS of 13 vs. 103 months) (log-rank $\mathrm{P}<0.001$, Figure $2 F$ ). Furthermore, we found LUSC patients with low SMP30 expression had significantly worse OS in both the early and late clinical stage subgroups (log-rank $\mathrm{P}<0.001$, Figure 2G,2H).

\section{Effect of SMP30 OE on proliferation of NSCLC A549 and H1299 cells}

To verify the potential antitumor actions of SMP3O in NSCLC, we first examined its expression in multiple NSCLC cell lines (Figure $3 A$ ) and established stable SMP30 overexpressing A549 and H1299 cell lines through use of the SMP30 LV. SMP30 expression was confirmed by Western blot (Figure 3B). Subsequently, we performed cell viability and colony formation assays to evaluate the role of SMP30 in A549 and H1299 cell proliferation. Compared with the NC group, we found SMP30 OE significantly decreased the A549 and $\mathrm{H} 1299$ cell viability determined by CCK-8 analysis $(\mathrm{P}<0.05$, Figure $3 C)$. This anti-proliferative effect was further validated by the colony formation assay, in which SMP30 OE significantly decreased the colony formation ability in A549 and H1299 cells $(\mathrm{P}<0.05$, Figure $3 D)$. We also used the EdU incorporation assay to analyze the role of $S M P 30$ on proliferation, and we found SMP30 OE remarkably decreased the EdU-positive cells compared with the NC group $(\mathrm{P}<0.05$, Figure $3 E)$. To further validate the effect of SMP30 OE on inhibiting NSCLC cell proliferation, we established H1299 cell xenograft in athymic nude mice and measured their tumor volumes. We found that all nude mice developed subcutaneous tumors, but the tumor volume in SMP30 OE group was clearly reduced compared with NC group (Figure $3 F$ ). Aberrant activation or expression of $c-M y c$ contributes to tumor progression. Western blot analyses showed that $S M P 30$ OE inhibited the expression of $c-M y c$ both in vitro and in vivo (Figure $3 B, 3 G$ ). We further examined cellcycle proteins with Western blot analysis and found that the level of CyclinD1 was dramatically decreased in the $S M P 30$ overexpressing groups both in vivo and in vitro
(Figure 3B,3G). However, other cell cycle-related proteins did not significantly change in these cell lines (Figure S2A). Taken together, the results revealed that $S M P 30$ had an antiproliferative action on NSCLC cells.

\section{Role of HDAC4 in SMP30-mediated NSCLC inbibition}

To further explore the underlying mechanism of SMP30 OE on inhibiting NSCLC proliferation, we measured the level of HDAC family members in A549 and H1299 cells among the NC and SMP30 overexpressing groups. Our results showed that $H D A C 4$ was downregulated after $S M P 30$ OE both in vivo and in vitro (Figure $4 A, 4 B$ ) while other members showed no significant changes (Figure $4 A, 4 B$, Figure S2B). To further explore the role of $H D A C 4$ in NSCLC, we measured its levels in several NSCLC cell lines (Figure $4 C$ ) and then established stable $H D A C 4$ overexpressing A549 and H1299 cell lines using the HDAC4 $\mathrm{LV}$, and HDAC4 expression was confirmed by Western blot (Figure $4 D$ ). Colony formation assay was performed to show that HDAC4 OE significantly increased colony formation ability of both cell lines (Figure $4 E$ ). These results were also confirmed by the EdU incorporation assay (Figure $4 F$ ) and furthermore, Western blot results showed that the levels of $c-M y c$ and CyclinD1 were elevated after HDAC4 OE in both the A549 and H1299 cell lines (Figure 4D).

\section{Involvement of HDAC4 in SMP30-mediated NSCLC suppression}

To further confirm the involvement of HDAC4 in SMP30mediated NSCLC repression, we upregulated HDAC4 by transfecting HDAC4 LV into SMP30 overexpressing H1299 cells. The OE of HDAC4 was verified by Western blot and had no effect on the levels of SMP30 protein (Figure $5 A)$. HDAC4 OE markedly reversed SMP30 OE-induced $c-M y c$ and CyclinD1 inhibition in NSCLC cells (Figure $5 A$ ). Moreover, we found HDAC4 OE partially reversed the repressed proliferative ability of H1299 cells overexpressing SMP30 (Figure 5B). These results were confirmed by the EdU incorporation assay (Figure $5 C$ ). Furthermore, the in vivo study showed that HDAC4 OE significantly increased the mean volume of tumors in the H1299 SMP30 overexpressed xenograft group (Figure 5D). Western blot analyses of subcutaneous tumors indicated that the $\mathrm{OE}$ of HDAC4 reversed the $c-M y c$ and CyclinD1 inhibition both in vivo and in vitro (Figure $5 A, 5 E$ ). Taken together, these results suggested that $S M P 30$-mediated NSCLC inhibition 


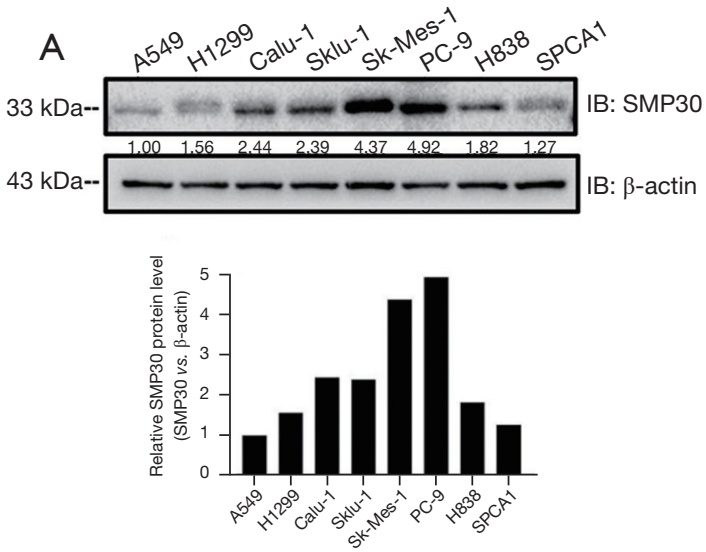

C

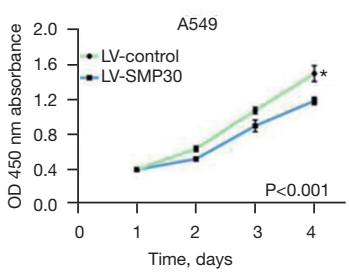

D
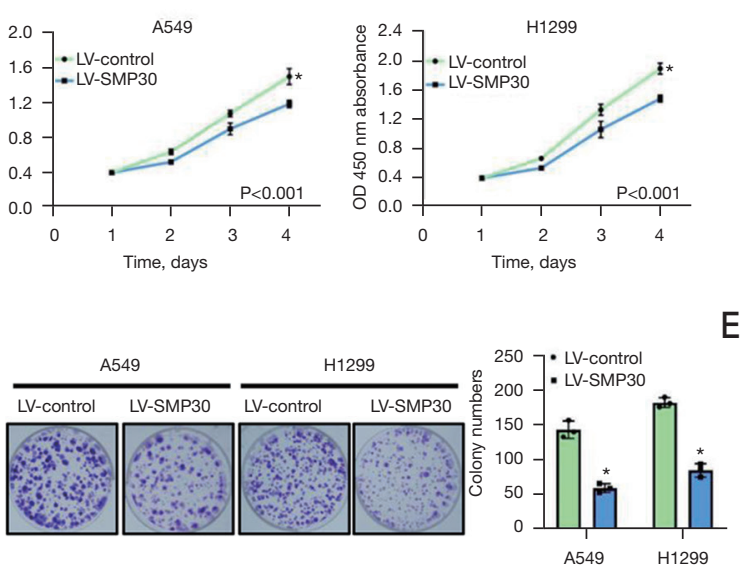

B

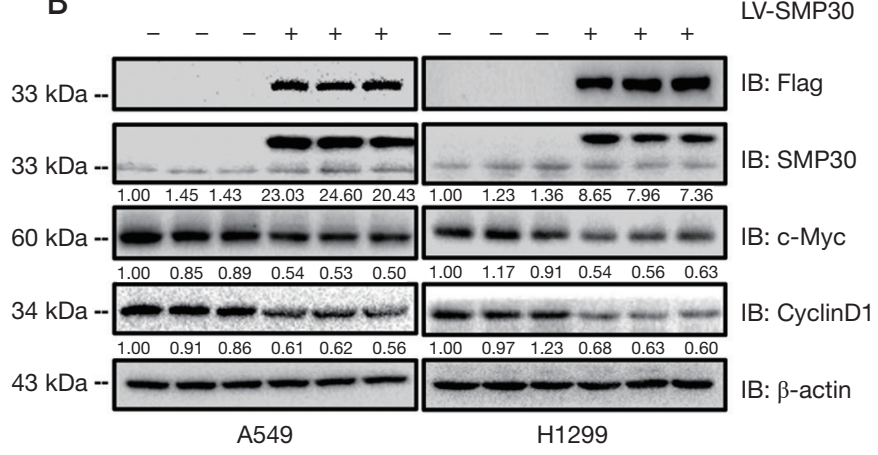

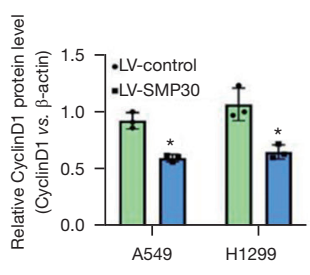

$\mathrm{E}$
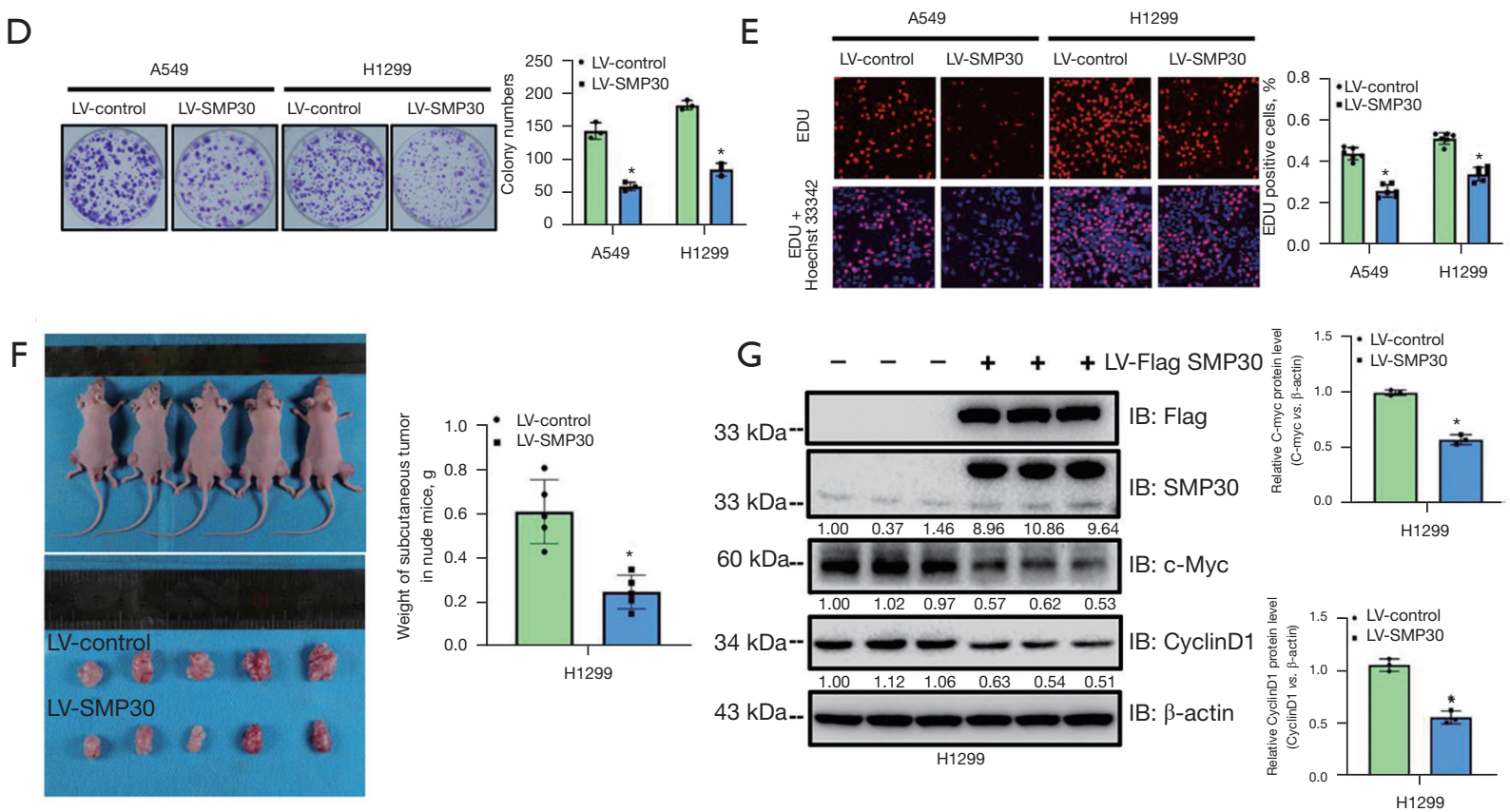

Figure 3 Effect of SMP30 overexpression on proliferative ability of A549 and H1299 cells, and tumor growth in H1299 cell tumor xenografts. (A) Relative protein levels of SMP30 in cell lines as determined by Western blot. $\beta$-actin was used as a loading control. (B) Representative Western blot results of Flag, SMP30, c-Myc and CyclinD1 in LV-SMP30 A549 and LV-SMP30 H1299 cells. (C) Growth curves of the A549 and H1299 cells. Cell viability was detected by CCK-8 assay and expressed as optical density values. (D) Representative images and results of the colony formation assay. Colonies were visualized by crystal violet staining. (E) Representative images $(\times 20)$ and statistical analysis of EdU incorporation assay. The results are presented as the ratio between the number of EdU-positive cells (red fluorescence) and the total number of Hoechst 33342-stained cells (blue fluorescence). (F) Representative results of tumor weight changes after subcutaneous injection of H1299 cells. Photographs show tumor xenograft morphologies in the LV-control and LV-SMP30 H1299 groups. (G) Representative Western blot results of Flag, SMP30, c-Myc and CyclinD1 in H1299 xenograft tumor tissues. $\beta$-actin was used as a Western blot loading control. The values below the Western blot band represent the relative gray values. All the data are shown as the mean $\pm \mathrm{SD}$. Student's $t$-test, ${ }^{*}, \mathrm{P}<0.05 v s$. LV-control group. LV, lentivirus; SMP30, senescence marker protein 30. 
A
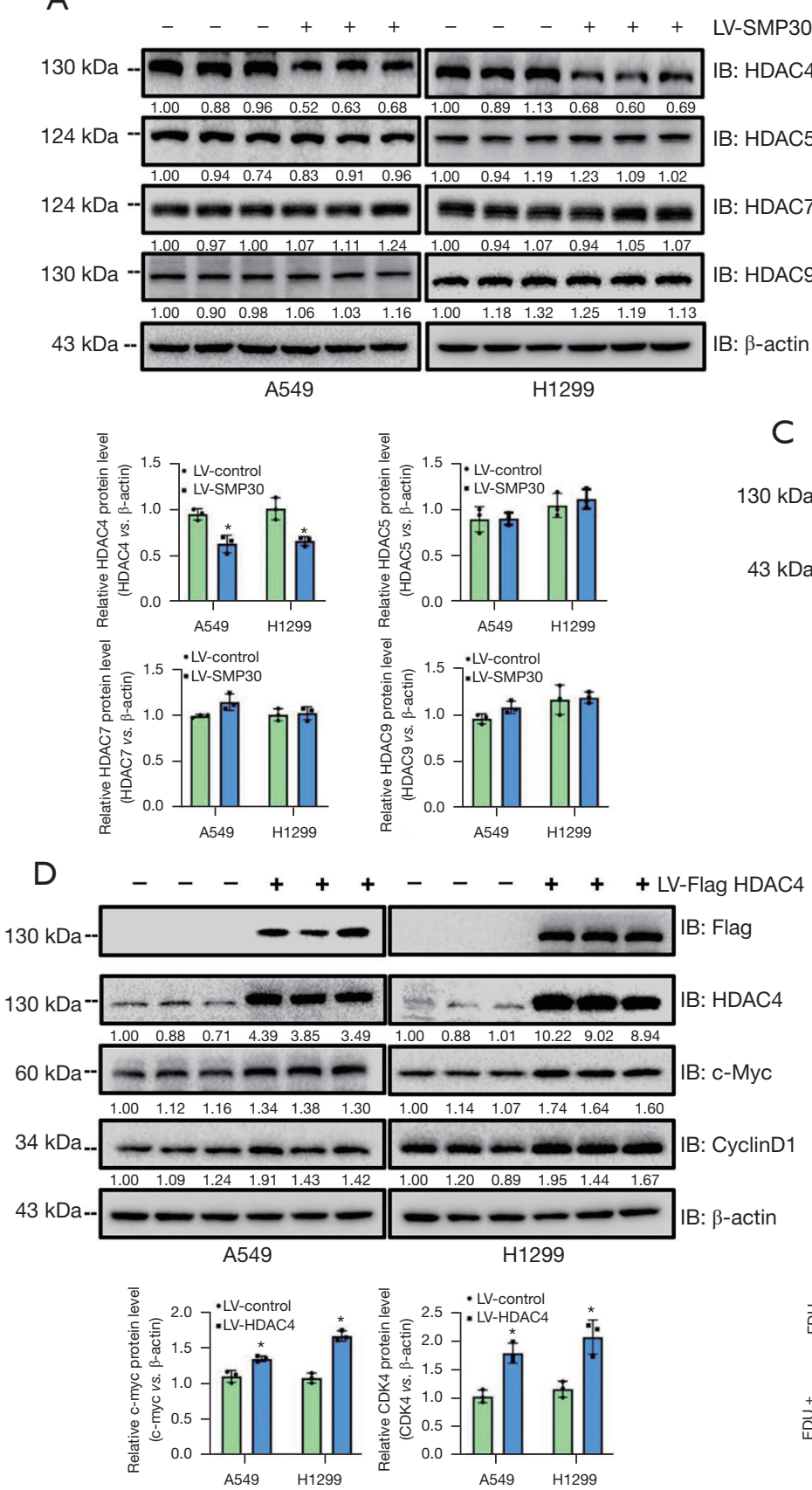

LV-SMP30

IB: HDAC4

IB: HDAC5

IB: HDAC7

IB: HDAC9

IB: $\beta$-actin
B

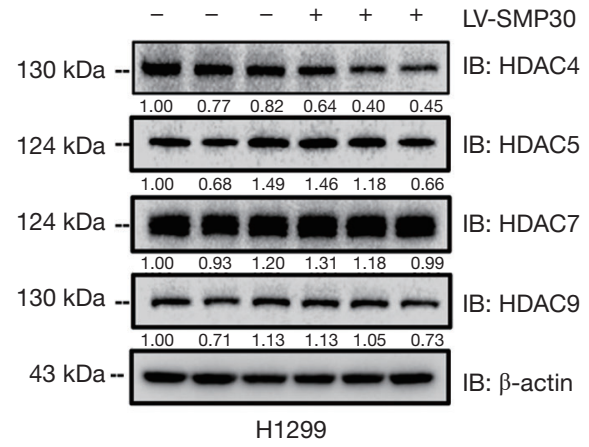

C

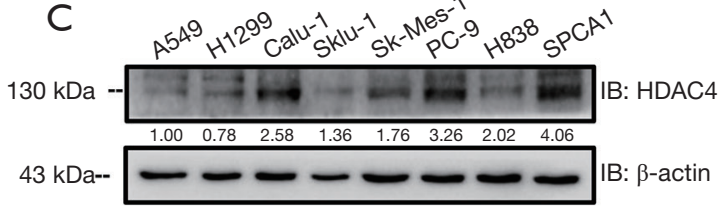

E
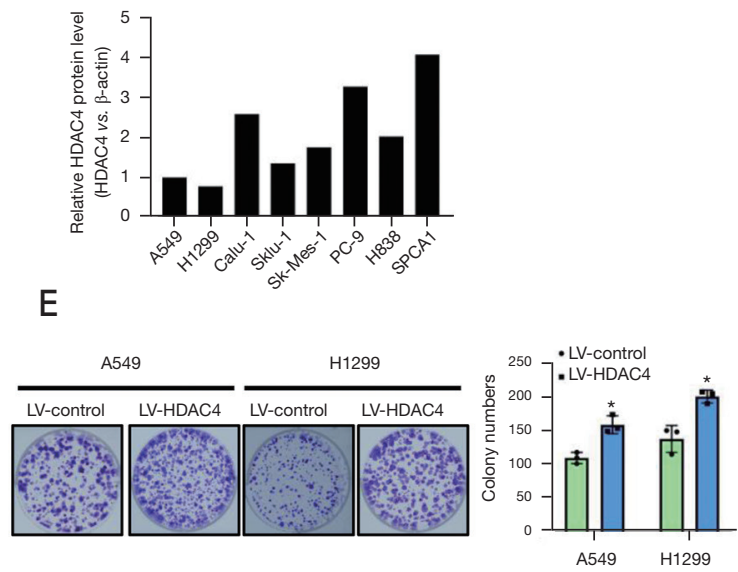

$\mathrm{F}$
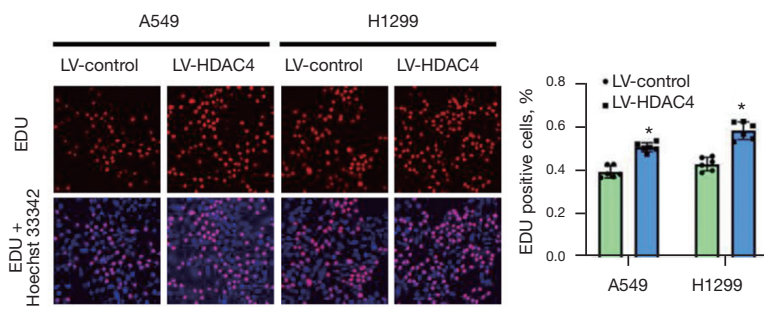

Figure 4 Effect of HDAC4 level of SMP30 overexpression both in vivo and in vitro, and effect of HDAC4 overexpression on NSCLC cell proliferation. (A) Representative Western blot results of HDAC4, HDAC5, HDAC7 and HDAC9 in LV-SMP30 A549 and LV-SMP30 H1299 cells. (B) Representative Western blot results of HDAC4, HDAC5, HDAC7 and HDAC9 in H1299 xenograft tumor tissues. (C) Relative protein levels of HDAC4 in cell lines as determined by Western blot. (D) Representative Western blot results of Flag, HDAC4, c-Myc and CyclinD1 in LVHDAC4 A549 and LV-HDAC4 H1299 cells. (E) Representative images and results of colony formation assay. Colonies were visualized by crystal violet staining. (F) Representative images ( $\times 20)$ and statistical analysis of EdU incorporation assay. The results are presented as the ratio between the number of EdU-positive cells (red fluorescence) and the total number of Hoechst 33342-stained cells (blue fluorescence). $\beta$-actin was used as a Western blot loading control. The values below the Western blot band represent the relative gray values. All the data are shown as the mean \pm SD. Student's $t$-test, *, $\mathrm{P}<0.05$ vs. LV-control group. HDAC histone deacetylase; LV, lentivirus; SMP30, senescence marker protein 30; SD, standard deviation; NSCLC, non-small cell lung cancer. 


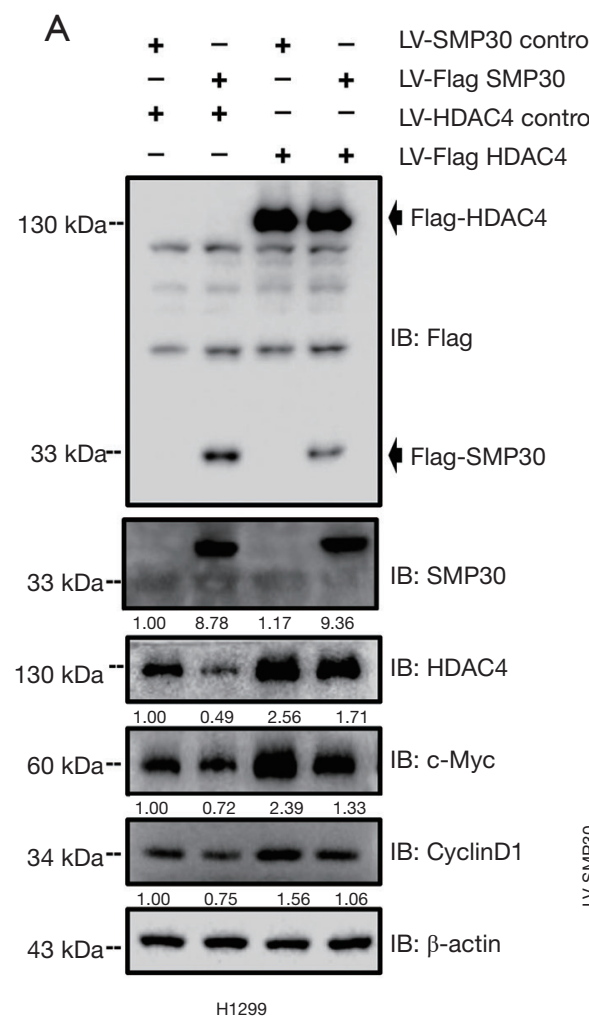

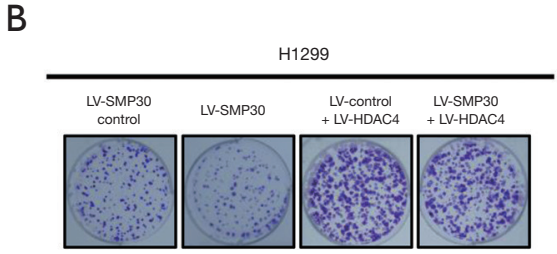

C
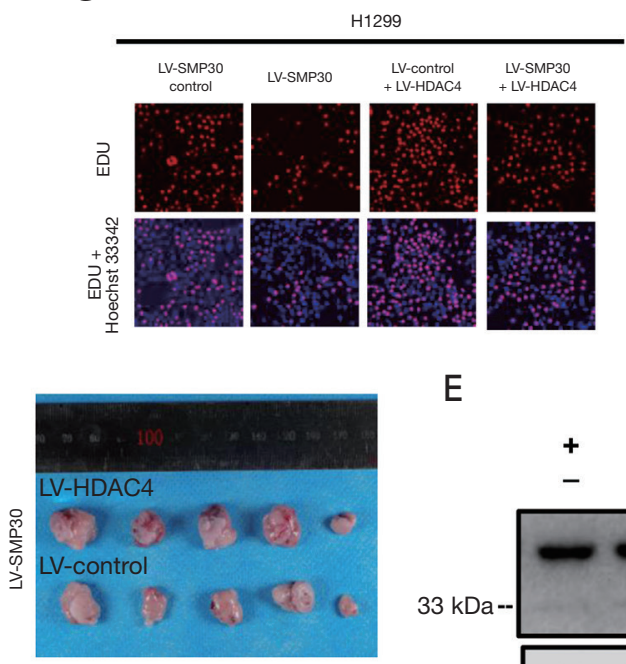

$\mathrm{E}$
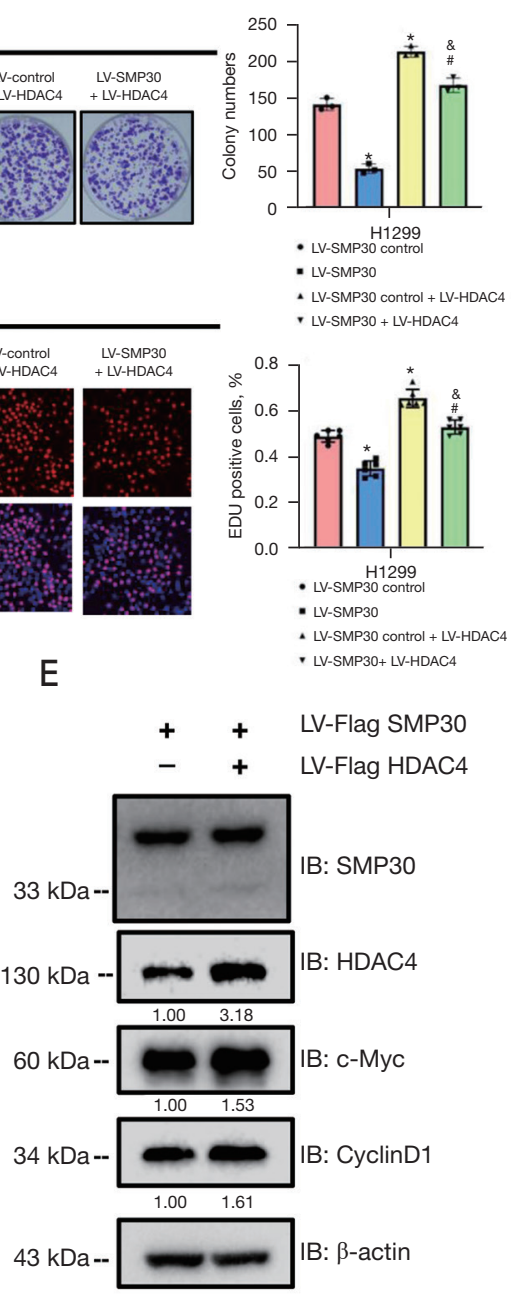

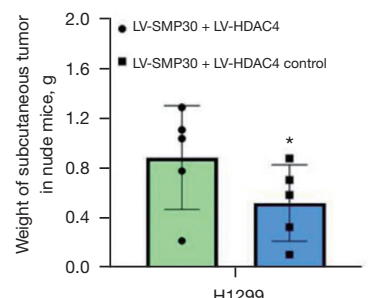

Figure 5 Essential requirement for HDAC4 in SMP30-mediated NSCLC cell proliferation. (A) Representative Western blot results of Flag, SMP30, HDAC4 and c-Myc and CyclinD1. (B) Representative images and results of colony formation assay. Colonies were visualized by crystal violet staining. (C) Representative images $(\times 20)$ and statistical analysis of EdU incorporation assay. The results are presented as the ratio between the number of EdU-positive cells (red fluorescence) and the total number of Hoechst 33342-stained cells (blue fluorescence). (D) Representative results for tumor weight changes after subcutaneous injection of H1299 cells. Photographs show tumor xenograft morphologies in the LV-SMP30 + LV-HDAC4 and LV-SMP30 + LV-control H1299 groups. (E) Representative Western blot results of SMP30, HDAC4, $c-M y c$ and CyclinD1 in H1299 xenograft tumor tissues. $\beta$-actin was used as a Western blot loading control. The values below the Western blot band represent the relative gray values. All the data are shown as the mean $\pm \mathrm{SD}$. Student's $t$-test, ${ }^{*}, \mathrm{P}<0.05 v s$. the LV-SMP30 control group; ", $\mathrm{P}<0.05$ vs. the LV-SMP30 group; ${ }^{\circ}, \mathrm{P}<0.05$ vs. the LV-SMP30 control + LV-HDAC4 group. HDAC, histone deacetylase; LV, lentivirus; SMP30, senescence marker protein 30; SD, standard deviation; NSCLC, non-small cell lung cancer.

can be reversed by HDAC4 upregulation.

\section{Effect of pharmacological inbibition of HDAC4 on proliferative abilities of $\mathrm{H} 1299$ cells}

To further confirm the role of HDAC4 in NSCLC, normal cells and HDAC4 overexpressing cells were incubated with the pan-inhibitor vorinostat (SAHA) and the specific class IIA inhibitor TMP269 respectively. Western blot results showed that, with increasing concentrations of SAHA and TMP269, the level of HDAC4 decreased (Figure 6A,6B). Consistently, the levels of $c-M y c$ and CyclinD1 were also 

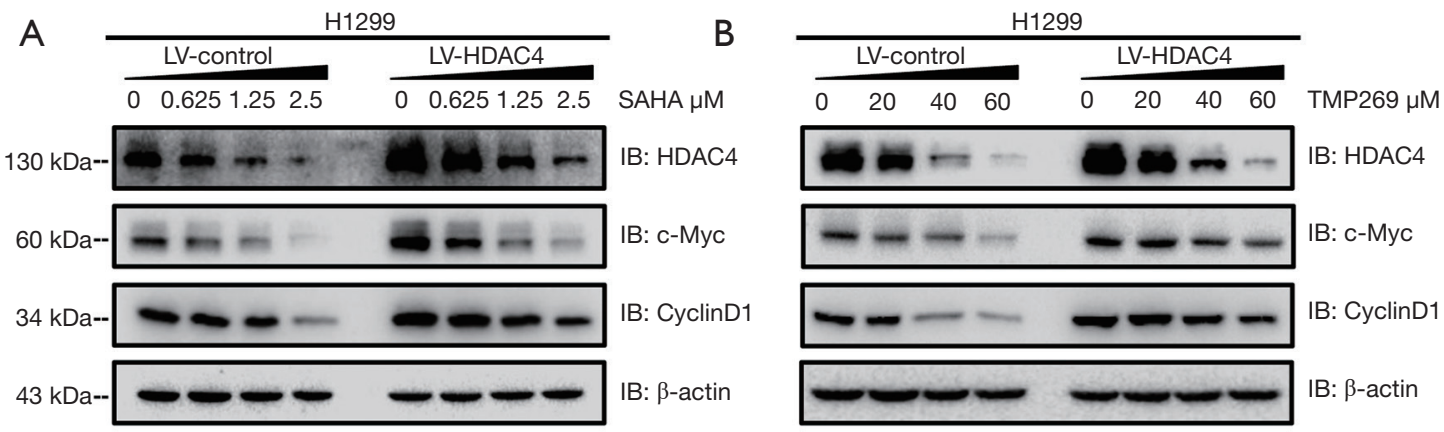

C

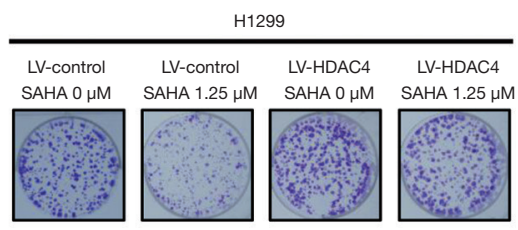

$\mathrm{D}$
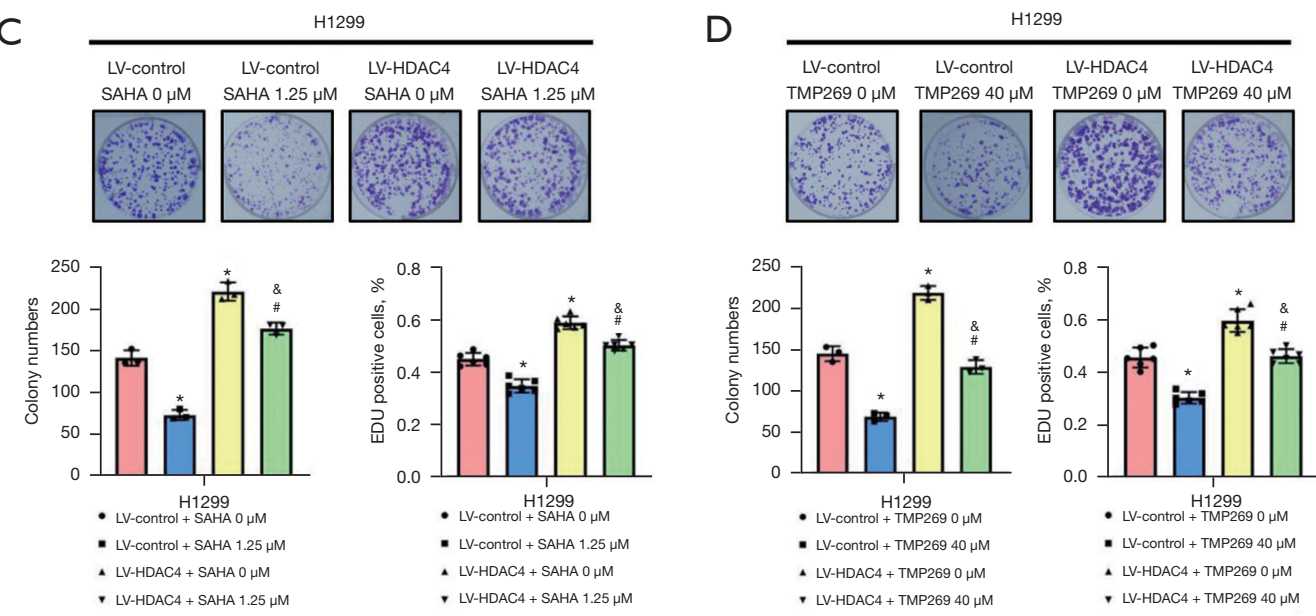

$\mathrm{E}$

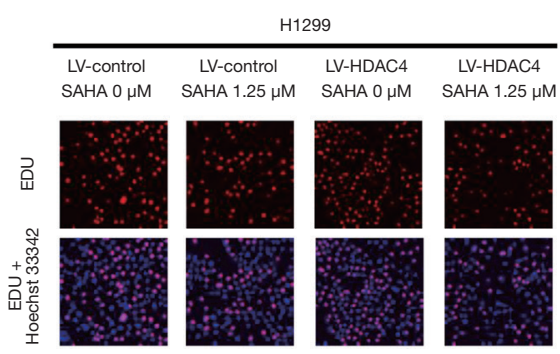

$\mathrm{F}$

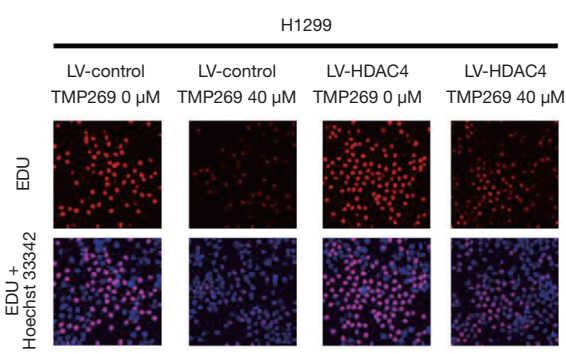

Figure 6 Effect of pharmacologic inhibition of HDAC4 on proliferative abilities of H1299 cells. LV-control and LV-HDAC4 H1299 cells were pretreated with SAHA or TMP269 for 48 h. (A,B) Representative Western blot results of HDAC4, c-Myc, and CyclinD1. (C,D) Representative images and results of colony formation assay. Colonies were visualized by crystal violet staining. (E,F) Representative images $(\times 20)$ and statistical analysis of EdU incorporation assay. The results are presented as the ratio between the number of EdU-positive cells (red fluorescence) and the total number of Hoechst 33342-stained cells (blue fluorescence). *, P $<0.05$ vs. the LV-control +SAHA/TMP269 $0 \mu M$

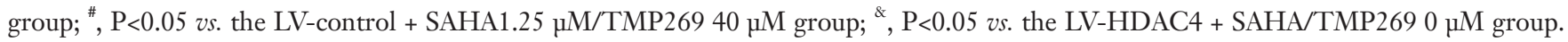
HDAC, histone deacetylase; LV, lentivirus; SMP30, senescence marker protein 30.

decreased and the average expressions of $H D A C 4, c-M y c$ and CyclinD1 in the HDAC4 overexpressing groups were significantly higher than in the normal cell groups (Figure $6 A, 6 B)$. Moreover, the colony formation assay showed that as the level of HDAC4 decreased, the cells' proliferative ability was inhibited (Figure 6C,6D). These results were confirmed by the EdU incorporation assay (Figure $6 E, 6 F$ ).

\section{Discussion}

SMP30, a multifunctional protein, plays a pivotal role in cell homeostasis (4), through maintaining calcium homeostasis, 
inhibiting various signaling pathways involving various protein kinases and protein phosphatases, suppressing nuclear DNA and RNA synthesis, and preventing cell proliferation and apoptosis (16,32). Furthermore, downregulation of SMP3O during aging might contribute to the deterioration of cellular functions and interactivity. It is well known that cancer is more common with aging (33). Previous studies suggest that $S M P 30$ is downregulated and exerts anticancer actions in human tumor tissues (15), but its oncostatic effects and detailed molecular mechanisms in NSCLC remain unclear. Consistent with previous research, our databases-mining results indicated that SMP30 mRNA levels were lower in NSCLC compared with normal lung tissues. Furthermore, that outcome was confirmed by our IHC analysis results based on tissue microarray containing 341 paired NSCLC tumor and normal samples. SMP30 expression negatively correlated with tumor size, tumor invasion, lymphatic metastasis, distant metastasis, cancer differentiation and clinical stage.

To the best of our knowledge, this study is the first to show a connection between SMP30 negativity and poor prognosis, and to indicate that $S M P 30$ may be an independent prognostic factor for NSCLC patients. NSCLC patients, both LUAD and LUSC with low SMP30 expression had a significantly shorter OS time than those with high expression. Furthermore, we also found that NSCLC patients with low SMP30 expression had poor prognosis regardless of stage at diagnosis. Interestingly, the Kaplan-Meier plotter database showed that low SMP30 expression was a predictor of poor prognosis in LUAD but not in LUSC patients, but our analysis demonstrated that low SMP30 level was associated with the poor prognosis of both LUAD and LUSC patients. We supposed that the differences may be due to that the Kaplan-Meier plotter database was based on mRNA and the expression of SMP3O in our study was based on IHC, which may be more reliable.

Several studies report that SMP30 OE inhibited cell proliferation in pancreatic cancer (34), colorectal carcinoma (17), liver cancer (18), and breast cancer (5) in vitro. Those findings support the idea that SMP30 may play an important inhibitory role in human cancer cells and that downregulation of SMP30 expression may lead to carcinogenesis. We found SMP30 OE significantly inhibited the proliferative ability (cell viability and colony formation assays) of A549 and H1299 cells. Additionally, the suppression of tumor growth by $S M P 30$ was further confirmed in a subcutaneous xenograft tumor nude mice model. Western blot analysis suggested that SMP30 OE downregulated the expression of HDAC4 both in vitro and in vivo.

HDAC4 is a member of the class IIa HDAC family and controls a complex cellular signaling cascade (19). It has diverse roles in cellular regulation, mainly including promoting chondrocyte differentiation (35), preventing neuronal death (36), promoting myocyte differentiation (37) and promoting the proliferation of satellite cells (38). Recently, numerous studies reported that OE of HDAC4 promoted cancer proliferation, invasion and metastasis via various signaling pathways, such as inhibition of $p 21$ and/or $p 27(20,22,24,39)$ or elevation of proliferating cell nuclear antigen $(P C N A)(40)$. These results indicate that $H D A C 4$ may act as a putative tumor promoter. In our study, the Western blot results showed that HDAC4 levels were significantly suppressed in $S M P 30$ overexpressing A549 and H1299 cell lines. Previous studies showed that pharmacological inhibition of HDAC suppressed the levels of $c-M y c$ and CyclinD1 $(41,42)$. Similarly, in our study, HDAC4 OE in the A549 and H1299 cell lines increased the levels of $c-M y c$ and $C y c l i n D 1$, and pharmacologic inhibition of $H D A C 4$ decreased both. Intriguingly, HDAC4 OE dramatically reversed the inhibition of SMP3O OE on cell proliferation both in vivo and in vitro but had no effect on the level of SMP30 expression.

Transcription factor $c-M y c$, product of gene $M Y C$, correlates with tumor aggression and poor clinical outcome in a variety of tumors (43). CyclinD1, encoded by CCND1 gene in humans, is an important regulator of the cell-cycle G1/S transition (44). $c-M y c$ and CyclinD1, which were often dysregulated and constitutively activated in human NSCLC, play important roles in many cancer types, suggesting that they may be key targets for treatment $(45,46)$. Previous studies report that $c-M y c$ increases the levels of CyclinD1, and further, activates $C D K 4$ and $C D K 6$, promoting G1phase cell-cycle progression (47). In this study, SMP30 OE significantly inhibited the levels of $c-M y c$ and $C y c l i n D 1$ both in vivo and in vitro, and this was dramatically reversed by $H D A C 4 \mathrm{OE}$. These results indicated that $S M P 30$ suppresses NSCLC proliferation via the $H D A C 4 / c-M y c$, CyclinD1 pathways.

In conclusion, we demonstrated for the first time that $S M P 30$ functions as a novel tumor suppressor factor in NSCLC. The level of SMP30 expression was significantly decreased in NSCLC tumors and a low level strongly correlated with worse OS of patients. Our findings indicated that SMP30 inhibited the development and progression of 
NSCLC through inhibiting cell proliferation via inhibition of HDAC4 expression. Therefore, targeting SMP30 may be a novel therapeutic strategy for NSCLC.

There are some limitations to this study that should be noted. Firstly, SMP30 was reported to be decreased during senescence (6) while SMP30 expression showed no significant changes with aging in our cohort, which may be due to our small size of data and deserve more efforts. Secondly, in the two parts: involvement of HDAC4 in SMP30-mediated NSCLC suppression and effect of pharmacological inhibition of HDAC4, we used the H1299 cell line nor the combination of A549 and H1299 cell lines, which may affect the reliability of our study and will be our subsequent work.

\section{Acknowledgments}

The authors appreciate the academic support from the AME Lung Cancer Collaborative Group.

Funding: This work was supported by the National Natural Science Foundation of China $(82103508,81871866$, 82173252), Shaanxi Special Support Plan-Program for Leading Talents of Science and Technology Innovation (No. 2019 Special Support Plan), the Natural Science Foundation of Shaanxi Province (2016SF-308, 2019SF033) and Project of Tangdu Hospital, the Fourth Military Medical University (No. 2018 Key Talents).

\section{Footnote}

Reporting Checklist: The authors have completed the ARRIVE reporting checklist. Available at https://dx.doi. org/10.21037/tlcr-21-982

Data Sharing Statement: Available at https://dx.doi. org/10.21037/tlcr-21-982

Conflicts of Interest: All authors have completed the ICMJE uniform disclosure form (available at https://dx.doi. org/10.21037/tlcr-21-982). TH reports that he has received personal fees from Chugai Pharmaceutical outside the submitted work. XY serves as an unpaid editorial board member of Translational Lung Cancer Research from July 2021 to June 2023. The other authors have no conflicts of interest to declare.

Ethical Statement: The authors are accountable for all aspects of the work in ensuring that questions related to the accuracy or integrity of any part of the work are appropriately investigated and resolved. All procedures performed in this study involving human participants were in accordance with the Declaration of Helsinki (as revised in 2013). This study was approved by the Ethics Committee of the Fourth Military Medical University (No. TDLL202110-03) and informed consents were not requested for this retrospective study. Animal experiments were performed under a project license (No. IACUC-20210609) granted by the Animal Ethics Committee of the Fourth Military Medical University, in compliance with the institutional guidelines for the care and use of animals.

Open Access Statement: This is an Open Access article distributed in accordance with the Creative Commons Attribution-NonCommercial-NoDerivs 4.0 International License (CC BY-NC-ND 4.0), which permits the noncommercial replication and distribution of the article with the strict proviso that no changes or edits are made and the original work is properly cited (including links to both the formal publication through the relevant DOI and the license). See: https://creativecommons.org/licenses/by-nc-nd/4.0/.

\section{References}

1. Siegel RL, Miller KD, Fuchs HE, et al. Cancer Statistics, 2021. CA Cancer J Clin 2021;71:7-33.

2. Monteverdi S, Vita E, Sartori G, et al. Long-term survivors with immunotherapy in ad-vanced NSCLC: is 'cure' within reach? Transl Cancer Res 2020;9:409-414.

3. Ganti AK, Klein AB, Cotarla I, et al. Update of Incidence, Prevalence, Survival, and Initial Treatment in Patients With Non-Small Cell Lung Cancer in the US. JAMA Oncol 2021;7:1824-32.

4. Yamaguchi M. Involvement of regucalcin as a suppressor protein in human carcinogenesis: insight into the gene therapy. J Cancer Res Clin Oncol 2015;141:1333-41.

5. Yamaguchi $M$, Osuka $S$, Weitzmann $M N$, et al. Increased regucalcin gene expression extends survival in breast cancer patients: Overexpression of regucalcin suppresses the proliferation and metastatic bone activity in MDAMB-231 human breast cancer cells in vitro. Int J Oncol 2016;49:812-22.

6. Scott SH, Bahnson BJ. Senescence Marker Protein 30: Functional and Structural Insights to its Unknown Physiological Function. Biomol Concepts 2011;2:469-80.

7. Fujisawa K, Terai S, Hirose $\mathrm{Y}$, et al. Senescence marker protein 30 (SMP30)/regucalcin (RGN) expression decreases 
with aging, acute liver injuries and tumors in zebrafish. Biochem Biophys Res Commun 2011;414:331-6.

8. Maruyama N, Ishigami A, Kondo Y. Pathophysiological significance of senescence marker protein-30. Geriatr Gerontol Int 2010;10 Suppl 1:S88-98.

9. Marques R, Maia CJ, Vaz C, et al. The diverse roles of calcium-binding protein regucalcin in cell biology: from tissue expression and signalling to disease. Cell Mol Life Sci 2014;71:93-111.

10. Misawa H, Inagaki S, Yamaguchi M. Suppression of cell proliferation and deoxyribonucleic acid synthesis in the cloned rat hepatoma H4-II-E cells overexpressing regucalcin. J Cell Biochem 2001;84:143-9.

11. Nakagawa T, Sawada N, Yamaguchi M. Overexpression of regucalcin suppresses cell proliferation of cloned normal rat kidney proximal tubular epithelial NRK52E cells. Int J Mol Med 2005;16:637-43.

12. Tsurusaki Y, Yamaguchi M. Suppressive role of endogenous regucalcin in the enhancement of deoxyribonucleic acid synthesis activity in the nucleus of regenerating rat liver. J Cell Biochem 2002;85:516-22.

13. Tsurusaki Y, Yamaguchi M. Overexpression of regucalcin modulates tumor-related gene expression in cloned rat hepatoma H4-II-E cells. J Cell Biochem 2003;90:619-26.

14. Yamaguchi M, Daimon Y. Overexpression of regucalcin suppresses cell proliferation in cloned rat hepatoma H4-II-E cells: involvement of intracellular signaling factors and cell cycle-related genes. J Cell Biochem 2005;95:1169-77.

15. Vaz CV, Correia S, Cardoso HJ, et al. The Emerging Role of Regucalcin as a Tumor Suppressor: Facts and Views. Curr Mol Med 2016;16:607-19.

16. Yamaguchi M, Osuka S, Hankinson O, et al. Prolonged survival of renal cancer patients is concomitant with a higher regucalcin gene expression in tumor tissues: Overexpression of regucalcin suppresses the growth of human renal cell carcinoma cells in vitro. Int J Oncol 2019;54:188-98.

17. Yamaguchi M, Osuka S, Murata T. Prolonged survival of patients with colorectal cancer is associated with a higher regucalcin gene expression: Overexpression of regucalcin suppresses the growth of human colorectal carcinoma cells in vitro. Int J Oncol 2018;53:1313-22.

18. Yamaguchi M, Murata T. Exogenous regucalcin suppresses the growth of human liver cancer HepG2 cells in vitro. Oncol Rep 2018;39:2924-30.

19. Mielcarek M, Zielonka D, Carnemolla A, et al. HDAC4 as a potential therapeutic target in neurodegenerative diseases: a summary of recent achievements. Front Cell Neurosci 2015;9:42.

20. Kang ZH, Wang CY, Zhang WL, et al. Histone deacetylase HDAC4 promotes gastric cancer SGC7901 cells progression via p21 repression. PLoS One 2014;9:e98894.

21. Zeng LS, Yang XZ, Wen YF, et al. Overexpressed HDAC4 is associated with poor survival and promotes tumor progression in esophageal carcinoma. Aging (Albany NY) 2016;8:1236-49.

22. Cai JY, Xu TT, Wang Y, et al. Histone deacetylase HDAC4 promotes the proliferation and invasion of glioma cells. Int J Oncol 2018;53:2758-68.

23. Zhou L, Xu X, Liu H, et al. Prognosis Analysis of Histone Deacetylases mRNA Expression in Ovarian Cancer Patients. J Cancer 2018;9:4547-55.

24. Cheng C, Yang J, Li SW, et al. HDAC4 promotes nasopharyngeal carcinoma progression and serves as a therapeutic target. Cell Death Dis 2021;12:137.

25. Feng S, Zhang L, Liu X, et al. Low levels of AMPK promote epithelial-mesenchymal transition in lung cancer primarily through HDAC4- and HDAC5mediated metabolic reprogramming. J Cell Mol Med 2020;24:7789-801.

26. Zhang X, Qi Z, Yin H, et al. Interaction between $\mathrm{p} 53$ and Ras signaling controls cisplatin resistance via HDAC4- and HIF-1 $\alpha$-mediated regulation of apoptosis and autophagy. Theranostics 2019;9:1096-114.

27. Zhang W, Peyton M, Xie Y, et al. Histone deacetylase inhibitor romidepsin enhances anti-tumor effect of erlotinib in non-small cell lung cancer (NSCLC) cell lines. J Thorac Oncol 2009;4:161-6.

28. Gray JE, Haura E, Chiappori A, et al. A phase I, pharmacokinetic, and pharmacodynamic study of panobinostat, an HDAC inhibitor, combined with erlotinib in patients with advanced aerodigestive tract tumors. Clin Cancer Res 2014;20:1644-55.

29. Wang L, Syn NL, Subhash VV, et al. Pan-HDAC inhibition by panobinostat mediates chemosensitization to carboplatin in non-small cell lung cancer via attenuation of EGFR signaling. Cancer Lett 2018;417:152-60.

30. Li W, Zheng G, Xia J, et al. Cell cycle-related and expression-elevated protein in tumor overexpression is associated with proliferation behaviors and poor prognosis in non-small-cell lung cancer. Cancer Sci 2018;109:1012-23.

31. Liu D, Ma Z, Di S, et al. AMPK/PGC1 $\alpha$ activation by melatonin attenuates acute doxorubicin cardiotoxicity via 
alleviating mitochondrial oxidative damage and apoptosis. Free Radic Biol Med 2018;129:59-72.

32. Yamaguchi M, Osuka S, Shoji M, et al. Survival of lung cancer patients is prolonged with higher regucalcin gene expression: suppressed proliferation of lung adenocarcinoma A549 cells in vitro. Mol Cell Biochem 2017;430:37-46.

33. Tidwell TR, Søreide K, Hagland HR. Aging, Metabolism, and Cancer Development: from Peto's Paradox to the Warburg Effect. Aging Dis 2017;8:662-76.

34. Yamaguchi M, Osuka S, Weitzmann MN, et al. Prolonged survival in pancreatic cancer patients with increased regucalcin gene expression: Overexpression of regucalcin suppresses the proliferation in human pancreatic cancer MIA PaCa-2 cells in vitro. Int J Oncol 2016;48:1955-64.

35. Guan Y, Chen Q, Yang X, et al. Subcellular relocation of histone deacetylase 4 regulates growth plate chondrocyte differentiation through $\mathrm{Ca} 2+/$ calmodulin-dependent kinase IV. Am J Physiol Cell Physiol 2012;303:C33-40.

36. Majdzadeh N, Wang L, Morrison BE, et al. HDAC4 inhibits cell-cycle progression and protects neurons from cell death. Dev Neurobiol 2008;68:1076-92.

37. Wei X, Li H, Zhang B, et al. miR-378a-3p promotes differentiation and inhibits proliferation of myoblasts by targeting HDAC4 in skeletal muscle development. RNA Biol 2016;13:1300-9.

38. Marroncelli N, Bianchi M, Bertin M, et al. HDAC4 regulates satellite cell proliferation and differentiation by targeting P21 and Sharp1 genes. Sci Rep 2018;8:3448.

Cite this article as: Shao C, Guo K, Xu L, Zhang Y, Duan H, Feng Y, Pan M, Lu D, Ren X, Ganti AK, Hakozaki T, Han J, Yan X, Ma Z. Senescence marker protein 30 inhibits tumor growth by reducing HDAC4 expression in non-small cell lung cancer. Transl Lung Cancer Res 2021;10(12):4558-4573. doi: $10.21037 /$ tlcr-21-982
39. Shen YF, Wei AM, Kou Q, et al. Histone deacetylase 4 increases progressive epithelial ovarian cancer cells via repression of p21 on fibrillar collagen matrices. Oncol Rep 2016;35:948-54.

40. Cao K, Wang H, Fang Y, et al. Histone Deacetylase 4 Promotes Osteosarcoma Cell Proliferation and Invasion by Regulating Expression of Proliferating Cell Nuclear Antigen. Front Oncol 2019;9:870.

41. Jin JS, Tsao TY, Sun PC, et al. SAHA inhibits the growth of colon tumors by decreasing histone deacetylase and the expression of cyclin D1 and survivin. Pathol Oncol Res 2012;18:713-20.

42. Nebbioso A, Carafa V, Conte M, et al. c-Myc Modulation and Acetylation Is a Key HDAC Inhibitor Target in Cancer. Clin Cancer Res 2017;23:2542-55.

43. Lin CY, Lovén J, Rahl PB, et al. Transcriptional amplification in tumor cells with elevated c-Myc. Cell 2012;151:56-67.

44. Qi Y, Wang D, Huang W, et al. CyclinD1 inhibits dicer and crucial miRNA expression by chromatin modification to promote the progression of intrahepatic cholangiocarcinoma. J Exp Clin Cancer Res 2019;38:413.

45. Venneti S, Thompson CB. Metabolic modulation of epigenetics in gliomas. Brain Pathol 2013;23:217-21.

46. Qu J, Li J, Zhang Y, et al. AKR1B10 promotes breast cancer cell proliferation and migration via the PI3K/AKT/ NF- $\kappa$ B signaling pathway. Cell Biosci 2021;11:163.

47. Bretones G, Delgado MD, León J. Myc and cell cycle control. Biochim Biophys Acta 2015;1849:506-16. 
(A)

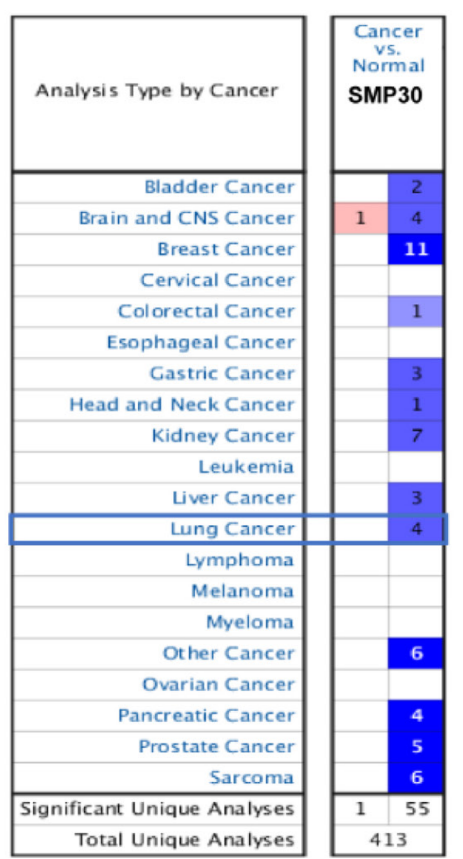

(B)
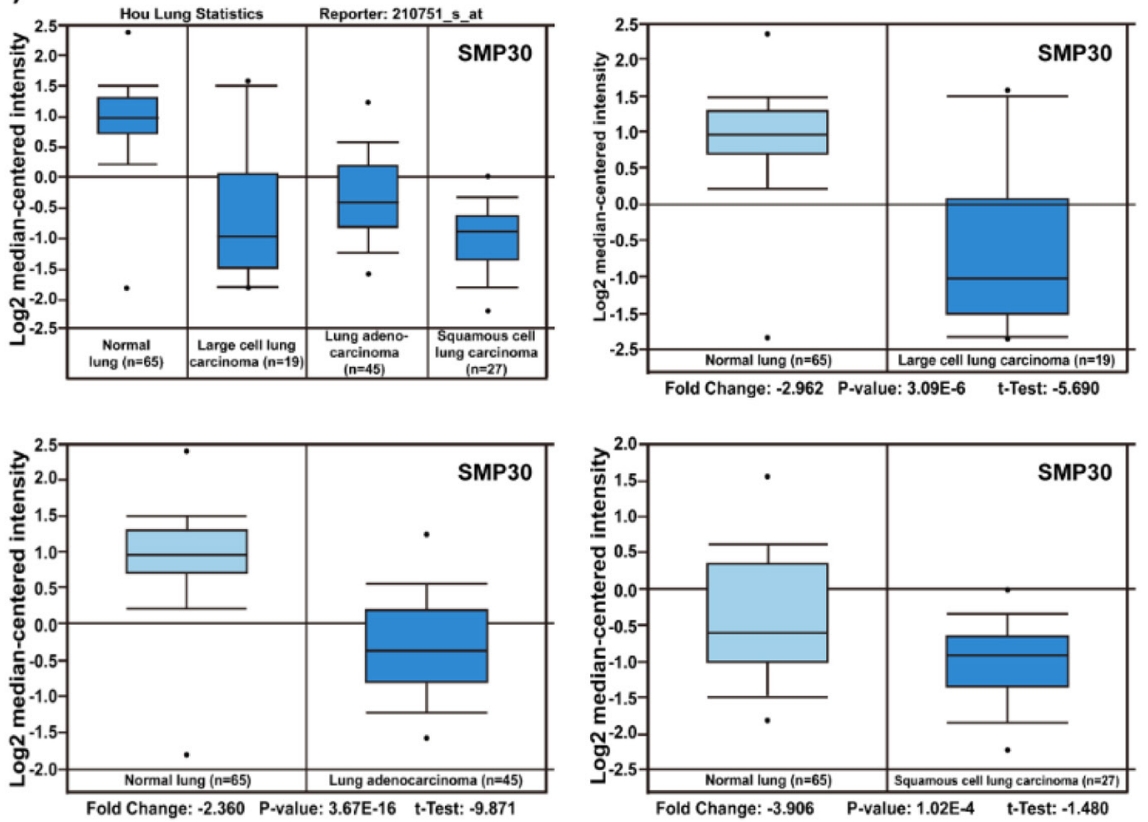

Figure S1 Downregulated SMP30 expression in NSCLC tumor tissues. (A) Number of Oncomine datasets with significant mRNA overexpression (red) or downregulated-expression (blue) of SMP30 (cancer vs. normal tissue). The P value threshold is 0.05. (B) SMP30 mRNA levels of NSCLC compared with the normal lung sample in Oncomine statistics. SMP30, senescence marker protein 30; NSCLC, non-small cell lung cancer.

(A)

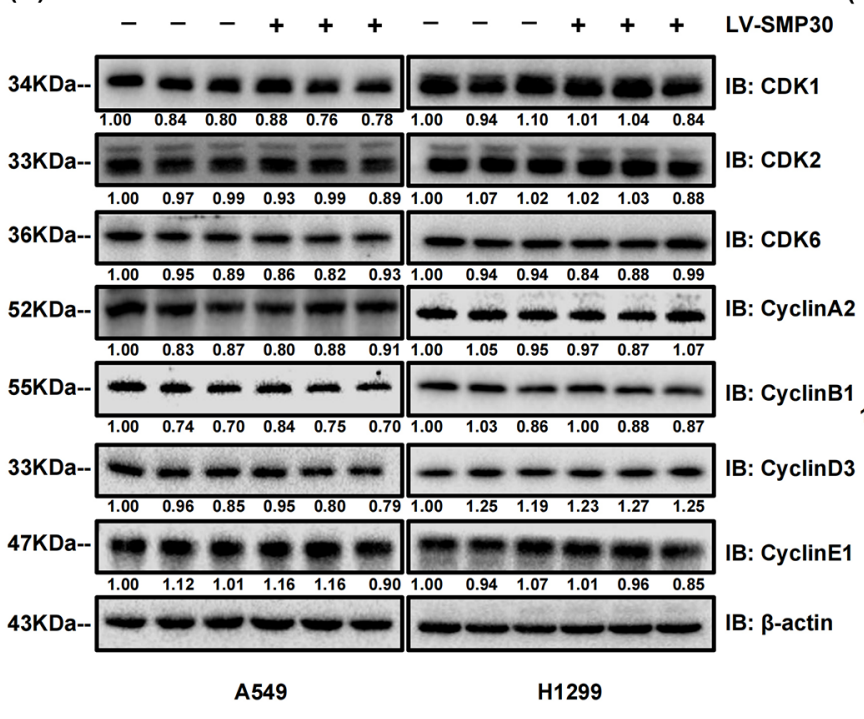

(B)
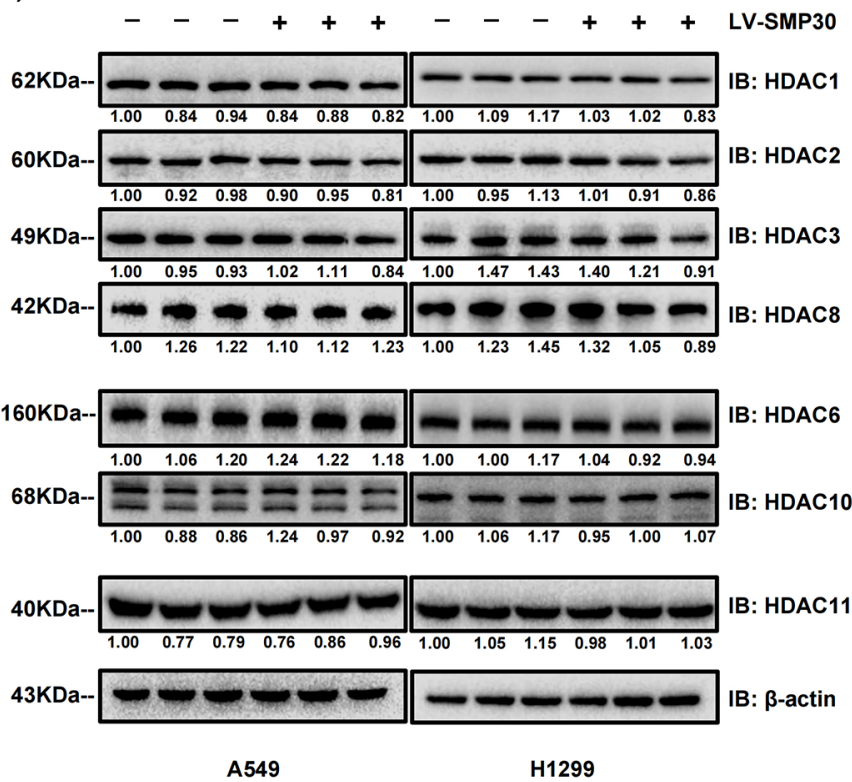

Figure S2 Western blot results of other members of cell cycle-related proteins and HDAC family. (A) Representative Western blot results of other cell cycle-related proteins in LV-SMP30 A549 and LV-SMP30 H1299 cells. (B) Representative Western blot results of other members of HDAC family in LV-SMP30 A549 and LV-SMP30 H1299 cells. HDAC, histone deacetylase; LV, lentivirus; SMP30, senescence marker protein 30. 